\title{
Search for top squark pair production in a final state with two tau leptons in proton-proton collisions at $\sqrt{s}=13 \mathrm{TeV}$
}

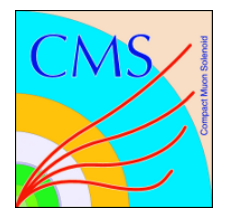

\section{The CMS collaboration}

E-mail: cms-publication-committee-chair@cern.ch

ABSTRACT: A search for pair production of the supersymmetric partner of the top quark, the top squark, in proton-proton collision events at $\sqrt{s}=13 \mathrm{TeV}$ is presented in a final state containing hadronically decaying tau leptons and large missing transverse momentum. This final state is highly sensitive to high- $\tan \beta$ or higgsino-like scenarios in which decays of electroweak gauginos to tau leptons are dominant. The search uses a data set corresponding to an integrated luminosity of $77.2 \mathrm{fb}^{-1}$, which was recorded with the CMS detector during 2016 and 2017. No significant excess is observed with respect to the background prediction. Exclusion limits at 95\% confidence level are presented in the top squark and lightest neutralino mass plane within the framework of simplified models, in which top squark masses up to $1100 \mathrm{GeV}$ are excluded for a nearly massless neutralino.

KEYwords: Hadron-Hadron scattering (experiments), Supersymmetry, Top physics

ArXIV EPRINT: 1910.12932 


\section{Contents}

1 Introduction 1

2 The CMS detector 3

3 Monte Carlo simulation 3

4 Event reconstruction $\quad 5$

$\begin{array}{lll}5 & \text { Event selection } & 7\end{array}$

$\begin{array}{llr}6 & \text { Background estimation } & \mathbf{8}\end{array}$

6.1 Tau lepton pairs from top production 8

6.2 Misidentified hadronically decaying tau lepton candidates 11

$\begin{array}{lll}7 & \text { Systematic uncertainties } & 13\end{array}$

8 Results $\quad 14$

$\begin{array}{lll}9 & \text { Summary } & 17\end{array}$

$\begin{array}{lr}\text { The CMS collaboration } & 25\end{array}$

\section{Introduction}

Supersymmetry (SUSY) [1-9] is one of the most widely studied theories of physics beyond the standard model (SM), providing solutions to various shortcomings of the SM. In SUSY models there is a bosonic superpartner for each fermion (and vice-versa), the superpartner having the same quantum numbers, other than spin, as its SM partner. The superpartners of the SM gauge and Higgs bosons (gauginos and higgsinos, respectively) mix to produce charginos and neutralinos. The weakly interacting lightest neutralino $\widetilde{\chi}_{1}^{0}$ can be a dark matter candidate in $R$-parity conserving SUSY models [10]. The SUSY partners of leftand right-handed top quarks are the top squarks, $\widetilde{t}_{L}$ and $\widetilde{t}_{R}$. These particles can mix with each other, resulting in physical states $\widetilde{t}_{1}$ and $\widetilde{t}_{2}$, with $\widetilde{t}_{1}$ defined to be the lighter of the two. The top squarks play an important role in stabilizing the Higgs boson mass by canceling the dominant top quark loop correction. Therefore, there is a strong motivation to perform searches for top squark production.

In this study, we focus on the signal of top squark pair production in a final state with two tau leptons. This probes the part of the parameter space of the minimal supersymmetric standard model (MSSM) in which the lightest charginos $\left(\widetilde{\chi}_{1}^{ \pm}\right)$and neutralino preferentially couple to third-generation fermions, such as tau leptons. The interaction of 

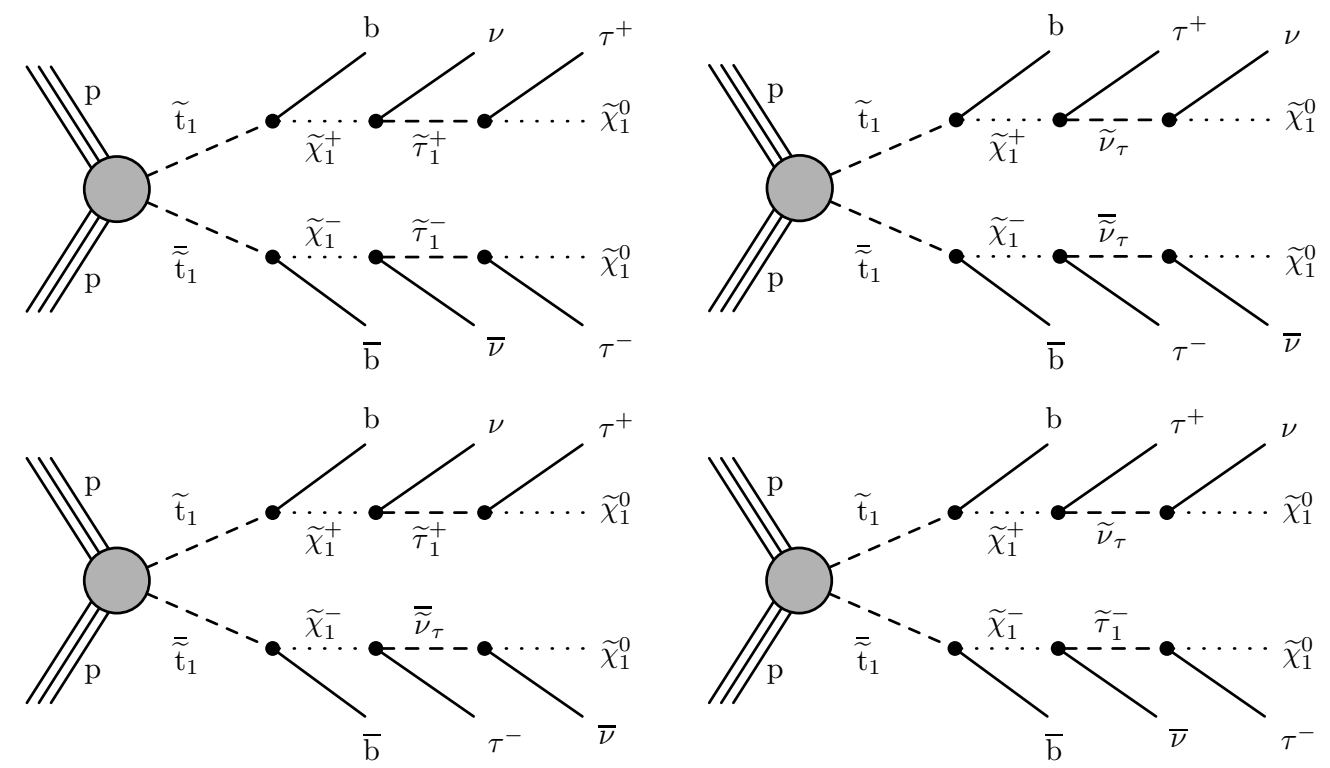

Figure 1. Top squark pair production in proton-proton collisions at the LHC, producing pairs of b quarks and taus accompanied by neutrinos and LSPs in the final state.

the charginos and neutralinos with fermion-sfermion pairs involves both gauge and Yukawa terms [9], so if charginos and neutralinos are predominantly higgsino-like, they will preferentially couple to third-generation fermion-sfermion pairs through the large Yukawa coupling. Moreover, the Yukawa coupling to the tau lepton-slepton pairs can be large for a high value of $\tan \beta$ even if the higgsino component is relatively small. Additionally, a large value of $\tan \beta$ can make the lighter state of the superpartner of the tau lepton $\left(\widetilde{\tau}_{1}\right)$ much lighter than the superpartners of the first and second generation leptons. Consequently, the chargino decays predominantly as $\tilde{\chi}_{1}^{+} \rightarrow \widetilde{\tau}_{1}^{+} v_{\tau}$ or $\tau^{+} \widetilde{v}_{\tau}$ (charge conjugation is assumed throughout in this paper), and the decay rates in the electron and muon channels are greatly reduced $[11,12]$. Therefore, searches for SUSY signals in electron and muon channels are less sensitive to this scenario.

We focus on the top squark decays $\tilde{\mathrm{t}}_{1} \rightarrow \mathrm{b} \tilde{\chi}_{1}^{+} \rightarrow \mathrm{b} \widetilde{\tau}_{1}^{+} v_{\tau} \rightarrow \mathrm{b} \tau^{+} \tilde{\chi}_{1}^{0} v_{\tau}$ and $\widetilde{\mathrm{t}}_{1} \rightarrow$ $\mathrm{b} \tilde{\chi}_{1}^{+} \rightarrow \mathrm{b} \tau^{+} \widetilde{v}_{\tau} \rightarrow \mathrm{b} \tau^{+} \tilde{\chi}_{1}^{0} v_{\tau}$. The $\tilde{\chi}_{1}^{0}$ is assumed to be the lightest SUSY particle (LSP). Being neutral and weakly interacting, it leaves no signature in the detector, resulting in an imbalance in transverse momentum $p_{\mathrm{T}}$. The neutrinos produced in the decay chains also contribute to the $p_{\mathrm{T}}$ imbalance. Hence, the events of interest contain two tau leptons, two b quarks, and a $p_{\mathrm{T}}$ imbalance. The decay chains are depicted by the four diagrams in figure 1 within the simplified model spectra (SMS) framework [13, 14]. It is assumed that the $\widetilde{\chi}_{1}^{+}$decays to $\widetilde{\tau}_{1}^{+}$or $\widetilde{v}_{\tau}$ with equal probability.

This search is performed using proton-proton collision events at a center-of-mass energy of $13 \mathrm{TeV}$, recorded by the CMS experiment at the CERN LHC. The data sample corresponds to integrated luminosities of 35.9 and $41.3 \mathrm{fb}^{-1}$ collected during the 2016 and 2017 operating periods of the LHC, respectively. Signal-like events are characterized by the presence of hadronically decaying tau leptons $\left(\tau_{\mathrm{h}}\right)$, jets identified as likely to have 
originated from the fragmentation of $\mathrm{b}$ quarks, and large missing $p_{\mathrm{T}}$. Contributions from SM processes with the same final state are estimated using a combination of Monte Carlo (MC) simulated samples and control samples in data.

Searches for top squark pair production in leptonic and hadronic final states have been performed by the CMS [15-22] and ATLAS [23-27] Collaborations, establishing limits on top squark masses in the framework of SMS models. The ATLAS Collaboration performed a search [28] based on 2015 and 2016 data probing the same final state as that used here, but optimized for a gauge-mediated SUSY breaking scenario with an almost massless gravitino as a source of missing momentum. Therefore, final states containing hadronically decaying tau leptons have not been extensively explored in the context of top squark searches motivated by high-tan $\beta$ and higgsino-like scenarios.

The paper is organized as follows. A brief description of the CMS detector is presented in section 2, followed by descriptions of the event simulation in section 3, and reconstruction in section 4 . The event selection and search strategy are detailed in section 5 . We explain the various methods used for background estimation in section 6 , the systematic uncertainties are discussed in section 7 , and the results are provided in section 8. Finally, the analysis is summarized in section 9.

\section{The CMS detector}

The central feature of the CMS apparatus is a superconducting solenoid of $6 \mathrm{~m}$ internal diameter, providing a magnetic field of $3.8 \mathrm{~T}$. Within the solenoid volume are a silicon pixel and strip tracker, a lead tungstate crystal electromagnetic calorimeter (ECAL), and a brass and scintillator hadron calorimeter (HCAL), each composed of a barrel and two endcap sections. Forward calorimeters extend the pseudorapidity coverage provided by the barrel and endcap detectors. Muons are detected in gas-ionization chambers embedded in the steel flux-return yoke outside the solenoid. A more detailed description of the CMS detector, together with a definition of the coordinate system used and the relevant kinematic variables, can be found in ref. [29].

Events of interest are selected using a two-tiered trigger system [30]. The first level, composed of custom hardware processors, uses information from the calorimeters and muon detectors to select events at a rate of around $100 \mathrm{kHz}$ within a time interval of less than $4 \mu \mathrm{s}$. The second level, known as the high-level trigger, consists of a farm of processors running a version of the full event reconstruction software optimized for fast processing, and reduces the event rate to around $1 \mathrm{kHz}$ before data storage.

\section{Monte Carlo simulation}

Simulation is used to estimate several of the SM backgrounds. The predictions for signal event rates are also estimated using simulation, based on simplified SUSY signal models. The simulation is corrected for small discrepancies observed with respect to collision data using a number of scale factors (SFs). These will be discussed in later sections. 
The pair production of top quarks $(\mathrm{t} \overline{\mathrm{t}})$ is generated at next-to-leading order (NLO) in $\alpha_{S}$ using POWHEG v2 [31-35]. The same POWHEG generator has been used for the single top quark $t$-channel process, whereas POWHEG v1 has been used for the tW process [36]. The MadGraph5_amc@NlO v2.2.2 (v2.4.2 for 2017) [37] generator is used at leading order (LO) for modeling the Drell-Yan+jets (DY+jets) and $\mathrm{W}+$ jets backgrounds, which are normalized to the next-to-next-to-leading order (NNLO) cross sections. The MADGRAPH5_aMC@NLO generator is also used at NLO for simulating the diboson, VH, and t $\overline{\mathrm{t}} \mathrm{V}$ $(\mathrm{V}=\mathrm{W}$ or $\mathrm{Z}$ ) backgrounds. For the 2016 analysis, the parton shower and hadronization are simulated with PYTHIA v8.212 [38] using the underlying event tunes CUETP8M2T4 [39]

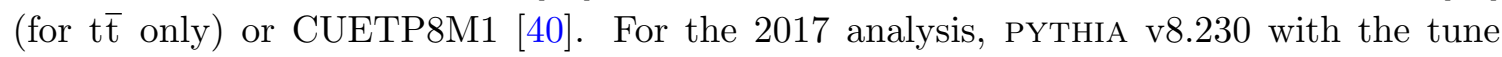
CP5 [41] is used. The CMS detector response is modeled using Geant4 [42], and the simulated events are then reconstructed in the same way as collision data.

Signal processes for top squark pair production shown in figure 1 are generated at LO using MAdGraPh5_amC@NLO v2.2.2. The tunes CUETP8M1 and CP2 [41] are used for the 2016 and 2017 analyses, respectively. The signal cross sections are evaluated using NNLO plus next-to-leading logarithmic (NLL) calculations [43-47]. Detector response for the signal events is simulated using the fast CMS detector simulation (FASTSiM) [48].

We assume a branching fraction of $50 \%$ for each of the two decay modes of the chargino, $\tilde{\chi}_{1}^{+} \rightarrow \widetilde{\tau}_{1}^{+} v_{\tau}$ and $\tilde{\chi}_{1}^{+} \rightarrow \tau^{+} \widetilde{v}_{\tau}$. Each of the four diagrams in figure 1 therefore contributes $25 \%$ of the generated signal events. The masses of SUSY particles appearing in the decay chain are determined by the parameterization

$$
\begin{aligned}
m_{\widetilde{\chi}_{1}^{-}}-m_{\widetilde{\chi}_{1}^{0}} & =0.5\left(m_{\widetilde{\mathrm{t}}_{1}}-m_{\widetilde{\chi}_{1}^{0}}\right), \\
m_{\widetilde{\tau}_{1}}-m_{\widetilde{\chi}_{1}^{0}} & =x\left(m_{\widetilde{\chi}_{1}^{-}}-m_{\widetilde{\chi}_{1}^{0}}\right), \\
x & \in\left[\begin{array}{ll}
0.25 & 0.5,0.75
\end{array}\right] \\
m_{\widetilde{v}_{\tau}} & =m_{\widetilde{\tau}_{1}} .
\end{aligned}
$$

In this parameterization, the chargino mass is fixed to be the mean of the top squark and $\tilde{\chi}_{1}^{0}$ masses. The masses of the leptonic superpartners are set by the value of $x$ for a given pair of top squark and $\widetilde{\chi}_{1}^{0}$ masses. The kinematic properties of the final state particles in each of the decay chains depicted in figure 1 therefore depend on the choice of $x$.

- $x=0.25$ : the mass of the lepton superpartner is closer to that of the $\widetilde{\chi}_{1}^{0}$ than to that of the $\tilde{\chi}_{1}^{-}$. Hence, the upper left diagram in figure 1 produces lower energy tau leptons than the upper right. The lower two diagrams both typically produce two tau leptons with a large difference in energy.

- $x=0.75$ : the masses of the $\widetilde{\tau}_{1}^{ \pm}$and the $\tilde{\chi}_{1}^{ \pm}$are relatively close, so the upper left diagram in figure 1 produces more energetic tau leptons than the upper right. The lower two diagrams produce the same energy asymmetry as in the case of $x=0.25$.

- $x=0.5$ : the tau leptons in all four diagrams have similar energies. 
In fact, when all four diagrams are taken into account the distributions of the kinematic properties are found to be very similar for the three different values of $x$, for a given set of chargino and LSP masses.

It is important to note, however, that the choice of chargino mass does affect the overall sensitivity. For instance, if the chargino is very close in mass to the top squark, then the momenta of the $\mathrm{b}$ jets are reduced and those of the remaining decay products are increased. This results in an increase in the overall sensitivity, provided the $\mathrm{b}$ jet $p_{\mathrm{T}}$ values are within the acceptance. On the other hand if the chargino is very close in mass to the LSP, then an overall loss of sensitivity is expected. Such scenarios are not explored in this paper, where the default chargino mass given in eq. (3.1) is taken throughout. The polarizations of the tau leptons originating from SUSY cascade decays, which have been found to be useful for studying SUSY signals [12], have not been exploited here.

\section{Event reconstruction}

The particle-flow (PF) algorithm [49] aims at reconstructing each individual particle in an event, with an optimized combination of information from the various components of the CMS detector. The energy of photons is obtained from the ECAL measurement, whereas the momentum of electrons is determined from a combination of the measurement of momentum by the tracker, the energy of matching ECAL deposits, and the energy of all bremsstrahlung photons consistent with originating from the track. The momentum of muons is obtained from the curvature of the corresponding track. The energy of charged hadrons is determined from a combination of the momentum measured in the tracker and the matching ECAL and HCAL energy deposits, corrected for zero-suppression effects and for the response function of the calorimeters to hadronic showers. Finally, the energy of neutral hadrons is obtained from the corresponding corrected ECAL and HCAL energies.

Reconstruction of jets is performed by clustering $\mathrm{PF}$ objects using the anti- $k_{\mathrm{T}}$ algorithm $[50,51]$ with a distance parameter of $R=0.4$. Jet momentum is determined as the vectorial sum of all particle momenta in the jet, and is found in simulation to be, on average, within $5-10 \%$ of the generated momentum over the whole $p_{\mathrm{T}}$ spectrum and detector acceptance. Additional proton-proton interactions within the same or nearby bunch crossings (pileup) can contribute spurious tracks and calorimetric energy deposits, increasing the apparent jet momentum. In order to mitigate this effect, tracks identified as originating from pileup vertices are discarded, and an offset is applied to correct for the remaining contributions [52]. Jets are calibrated using both simulation and data studies [52]. Additional selection criteria are applied to each jet to remove those potentially dominated by instrumental effects or reconstruction failures [53]. Jets with $p_{\mathrm{T}}>20 \mathrm{GeV}$ and $|\eta|<2.4$ are used in this analysis.

Vertices reconstructed in an event are required to be within $24 \mathrm{~cm}$ of the center of the detector in the $z$ direction, and to have a transverse displacement from the beam line of less than $2 \mathrm{~cm}$. The vertex with the largest value of summed physics-object $p_{\mathrm{T}}^{2}$ is taken to be the primary pp interaction vertex. The physics objects used for this purpose are jets, clustered using the aforementioned jet finding algorithm with the tracks assigned to the 
vertex as inputs, and the associated missing transverse momentum, taken as the negative vector sum of the $p_{\mathrm{T}}$ of those jets.

Jets originating from the fragmentation of $\mathrm{b}$ quarks are identified as b-tagged jets by using the combined secondary vertex (CSVv2) algorithm [54], which utilizes information from displaced tracks and reconstructed secondary vertices. An operating point is chosen corresponding to a signal efficiency of $70 \%$ with a mistagging probability of about $1 \%$ for light jets (from up, down and strange quarks, and gluons) and $15 \%$ for jets originating from charm quarks.

The momentum resolution for electrons with $p_{\mathrm{T}} \approx 45 \mathrm{GeV}$ from $\mathrm{Z} \rightarrow$ ee decays ranges from 1.7 to $4.5 \%$. It is generally better in the barrel region than in the endcaps, and also depends on the bremsstrahlung energy emitted by the electron as it traverses the material in front of the ECAL [55]. Electrons with $p_{\mathrm{T}}>20 \mathrm{GeV}$ and $|\eta|<2.4$ are used for this analysis.

Muons are measured with detection planes made using three technologies: drift tubes, cathode strip chambers, and resistive plate chambers. Matching muons to tracks measured in the silicon tracker results in a $p_{\mathrm{T}}$ resolution of $1 \%$ in the barrel and $3 \%$ in the endcaps, for muons with a $p_{\mathrm{T}}$ of up to $100 \mathrm{GeV}$. The $p_{\mathrm{T}}$ resolution in the barrel is better than $7 \%$ for muons with a $p_{\mathrm{T}}$ of up to $1 \mathrm{TeV}$ [56]. This search uses muons with $p_{\mathrm{T}}>20 \mathrm{GeV}$ and $|\eta|<2.4$.

Isolation criteria are imposed on the lepton (electron and muon) candidates to reject leptons originating from hadronic decays. The isolation variable used for this purpose is defined as the scalar sum of the $p_{\mathrm{T}}$ of reconstructed charged and neutral particles within a cone of radius $\Delta R=\sqrt{(\Delta \eta)^{2}+(\Delta \phi)^{2}}=0.3(0.4)$ around the electron (muon) candidate track, excluding the lepton candidate, divided by the $p_{\mathrm{T}}$ of the lepton candidate. Charged particles not originating from the primary vertex are excluded from this sum and a correction is applied to account for the neutral components originating from pileup, following the procedure described in ref. [55]. This relative isolation is required to be less than 15 (20)\% for electron (muons). The electron and muon candidates passing the aforementioned criteria are used to identify a control region (CR) that is used for the estimation of the background from top quark pair production, as explained in section 6.1.

The missing transverse momentum vector $\vec{p}_{\mathrm{T}}^{\text {miss }}$ is computed as the negative vector sum of the $p_{\mathrm{T}}$ of all the PF candidates in an event, and its magnitude is denoted as $p_{\mathrm{T}}^{\text {miss }}$. The $\vec{p}_{\mathrm{T}}^{\text {miss }}$ is modified to account for the energy calibration of the reconstructed jets in the event. The energy calibration of the PF candidates that have not been clustered into jets is also taken into account. Anomalous high- $p_{\mathrm{T}}^{\text {miss }}$ events may appear because of a variety of reconstruction failures, detector malfunctions, or backgrounds not originating from collisions (e.g., particles in the beam halo). Such events are rejected by filters that are designed to identify more than $85-90 \%$ of the spurious high- $p_{\mathrm{T}}^{\text {miss }}$ events with a misidentification rate of less than $0.1 \%$ [57]. In order to minimize the effect of extra noise in the ECAL endcap in 2017 , forward jets with uncalibrated $p_{\mathrm{T}}<50 \mathrm{GeV}$ and $2.65<|\eta|<3.14$ are removed from the calculation of $p_{\mathrm{T}}^{\text {miss }}$ in both data and simulation. This improves the agreement between simulation and data at the cost of degrading the $p_{\mathrm{T}}^{\text {miss }}$ resolution by only a few percent.

The hadrons-plus-strips algorithm [58] is used to reconstruct $\tau_{\mathrm{h}}$ candidates: one charged hadron and up to two neutral pions, or three charged hadrons, consistent with 
originating from the decay of a tau lepton. The probability of an electron or muon being misidentified as a $\tau_{\mathrm{h}}$ candidate is greatly reduced by combining information from the tracker, calorimeters, and muon detector. The isolation of the $\tau_{\mathrm{h}}$ candidate is determined from the presence of reconstructed particles within a radius of $\Delta R=0.3$ around the $\tau_{\mathrm{h}}$ axis that are not compatible with the decay, and is a useful quantity to distinguish between jets and $\tau_{\mathrm{h}}$ decays. In order to distinguish between jets originating from quarks or gluons, and genuine hadronic tau lepton decays, a multivariate discriminant is calculated from information including the isolation and measured lifetime. The $\tau_{\mathrm{h}}$ candidates are selected with $p_{\mathrm{T}}>40 \mathrm{GeV},|\eta|<2.1$, and the "tight" working point of the above discriminant. This working point has an efficiency of $\approx 50 \%$ with a misidentification probability of $\approx 0.03 \%$. The "loose" working point, which has an efficiency of $\approx 65 \%$ and a misidentification probability of $\approx 0.07 \%$, is used for estimating the background from misidentified $\tau_{\mathrm{h}}$ candidates.

\section{Event selection}

The sources of $p_{\mathrm{T}}^{\text {miss }}$ in the signal events are the neutrinos and the weakly interacting neutralinos, which are correlated with the visible objects (in particular the $\tau_{\mathrm{h}}$ decays). In contrast, $p_{\mathrm{T}}^{\mathrm{miss}}$ in the SM background processes is primarily due to neutrinos. This difference can be exploited by first constructing the transverse mass $m_{\mathrm{T}}$, defined as follows:

$$
\begin{aligned}
m_{\mathrm{T}}^{2}\left({\overrightarrow{p_{\mathrm{T}}}}^{\mathrm{vis}}, \overrightarrow{p_{\mathrm{T}}}\right. & =m_{\text {vis }}^{2}+m_{\text {inv }}^{2}+2\left(E_{\mathrm{T}}^{\mathrm{vis}} E_{\mathrm{T}}^{\text {inv }}-{\overrightarrow{p_{\mathrm{T}}}}^{\text {vis }} \cdot{\overrightarrow{p_{\mathrm{T}}}}^{\text {inv }}\right), \\
\text { where } \quad E_{\mathrm{T}}^{2} & =m^{2}+p_{\mathrm{T}}^{2} .
\end{aligned}
$$

Here the masses of the visible (vis) and invisible (inv) particles are denoted by $m_{\mathrm{vis}}$ and $m_{\text {inv }}$, respectively. The value of $m_{\mathrm{T}}$ has a maximum at the mass of the parent of the visible and the invisible particles. To account for multiple sources of missing momentum in the signal process, the "stransverse mass" $[59,60]$ is defined as:

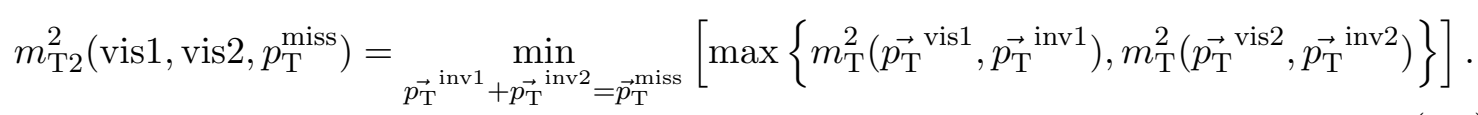

Since the momenta of the individual invisible particles in eq. (5.2) are unknown, $\vec{p}_{\mathrm{T}}^{\mathrm{miss}}$ is divided into two components $\left(\overrightarrow{p_{\mathrm{T}}}\right.$ inv1 and $\left.\overrightarrow{p_{\mathrm{T}}}{ }^{\text {inv2 }}\right)$ in such a way that the value of $m_{\mathrm{T} 2}$ is minimized. If $m_{\mathrm{T} 2}$ is computed using the two $\tau_{\mathrm{h}}$ candidates as the visible objects, vis1 and vis2, then its upper limit in the signal will be at the chargino mass. This is different from the SM background processes. For example in $t \bar{t}$ events, the upper limit is at the $\mathrm{W}$ boson mass. For this analysis, $m_{\mathrm{T} 2}$ is calculated with the masses of the invisible particles in eq. (5.1) set to zero [61].

The signal and background processes can be further separated by utilizing the total visible momentum of the system. This is characterized using the quantity $H_{\mathrm{T}}$, which is defined as the scalar sum of the $p_{\mathrm{T}}$ of all jets and the $\tau_{\mathrm{h}}$ candidates in the event. Jets lying within a cone of $\Delta R=0.3$ around either of the two selected $\tau_{\mathrm{h}}$ candidates are excluded from this sum to avoid double counting. Being a measure of the total energy of the system, $H_{\mathrm{T}}$ is sensitive to the mass of the top squark. 
Signal events are selected using $\tau_{\mathrm{h}} \tau_{\mathrm{h}}$ triggers, where both $\tau_{\mathrm{h}}$ candidates are required to have $|\eta|<2.1$, and $p_{\mathrm{T}}>35$ or $40 \mathrm{GeV}$ depending on the trigger path. The $\tau_{\mathrm{h}} \tau_{\mathrm{h}}$ trigger has an efficiency of $\approx 95 \%$ for $\tau_{\mathrm{h}}$ candidates that pass the offline selection. The trigger efficiencies in simulation are corrected to match the efficiencies measured in data. For the offline selection, signal events are required to have $p_{\mathrm{T}}^{\text {miss }}>50 \mathrm{GeV}, H_{\mathrm{T}}>100 \mathrm{GeV}$, at least two oppositely charged $\tau_{\mathrm{h}}$ candidates with $p_{\mathrm{T}}>40 \mathrm{GeV}$ and $|\eta|<2.1$, and at least one b-tagged jet with $p_{\mathrm{T}}>20 \mathrm{GeV}$ and $|\eta|<2.4$. The requirements on $p_{\mathrm{T}}^{\text {miss }}$ and the number of b-tagged jets $\left(n_{\mathrm{b}}\right)$ help to reduce the contributions from DY+jets and SM events comprised uniquely of jets produced through the strong interaction, referred to as multijet events. Distributions of the variables $p_{\mathrm{T}}^{\text {miss }}, m_{\mathrm{T} 2}$, and $H_{\mathrm{T}}$ after this selection are shown in figure 2 for data and the predicted background, along with representative signal distributions. The background prediction includes $t \bar{t}, D Y+$ jets, events with misidentified $\tau_{\mathrm{h}}$, and other rare SM processes. Detailed descriptions of the background estimation methods are presented in section 6 .

Signal events with different top squark and LSP masses populate different regions of the phase space. For example, regions with low $p_{\mathrm{T}}^{\text {miss }}, m_{\mathrm{T} 2}$, and $H_{\mathrm{T}}$ are sensitive to signals with low top squark masses. On the other hand, events with high $p_{\mathrm{T}}^{\text {miss }}, m_{\mathrm{T} 2}$, and $H_{\mathrm{T}}$ are sensitive to models with high top squark and low LSP masses. In order to obtain the highest sensitivity over the entire phase space, the signal region (SR) is divided into 15

bins as a function of the measured $p_{\mathrm{T}}^{\text {miss }}, m_{\mathrm{T} 2}$, and $H_{\mathrm{T}}$, which are illustrated in figure 3 .

\section{Background estimation}

The most significant background is t $\bar{t}$ production, either with two genuine $\tau_{\mathrm{h}}$ decays or because of jets being misidentified as $\tau_{\mathrm{h}}$ candidates. Because of theoretical uncertainties in the tE background modeling in the SR (which contains events that populate the tails of the kinematic distributions), we estimate the $t \overline{\mathrm{t}}$ contribution to events with two genuine $\tau_{\mathrm{h}}$ decays using CRs in data, as discussed below. The background contribution from DY events is typically minor in the most sensitive bins, and has been estimated using simulation. To account for residual discrepancies between data and the LO DY sample, correction factors for simulated events are derived from DY-enriched dimuon CRs in data and simulation as functions of the dimuon invariant mass and $p_{\mathrm{T}}$. The contribution from multijet events is negligible because of the selections $p_{\mathrm{T}}^{\text {miss }}>50 \mathrm{GeV}$ and $n_{\mathrm{b}} \geq 1$. Other less significant backgrounds, such as $\mathrm{W}+$ jets, $\mathrm{VV}, \mathrm{VH}$, and $\mathrm{t} \overline{\mathrm{t}} \mathrm{V}$ are also estimated from simulation. The overall SM contribution from jets being misidentified as $\tau_{\mathrm{h}}$ candidates is estimated using CRs in data. In the following sections we detail the estimation of those backgrounds that are obtained from CRs in data.

\subsection{Tau lepton pairs from top production}

The estimation of the background from $t \bar{t}$ events in which there are two genuine $\tau_{h}$ decays is based on the method described in ref. [62]. The predicted yields in each SR bin from simulation are multiplied by correction factors derived in a $t \bar{t}$-enriched $\mathrm{CR}$. The $\mathrm{t} \overline{\mathrm{t}}$-enriched $\mathrm{CR}$ is identified by selecting events with an oppositely charged e $\mu$ pair. These events are 

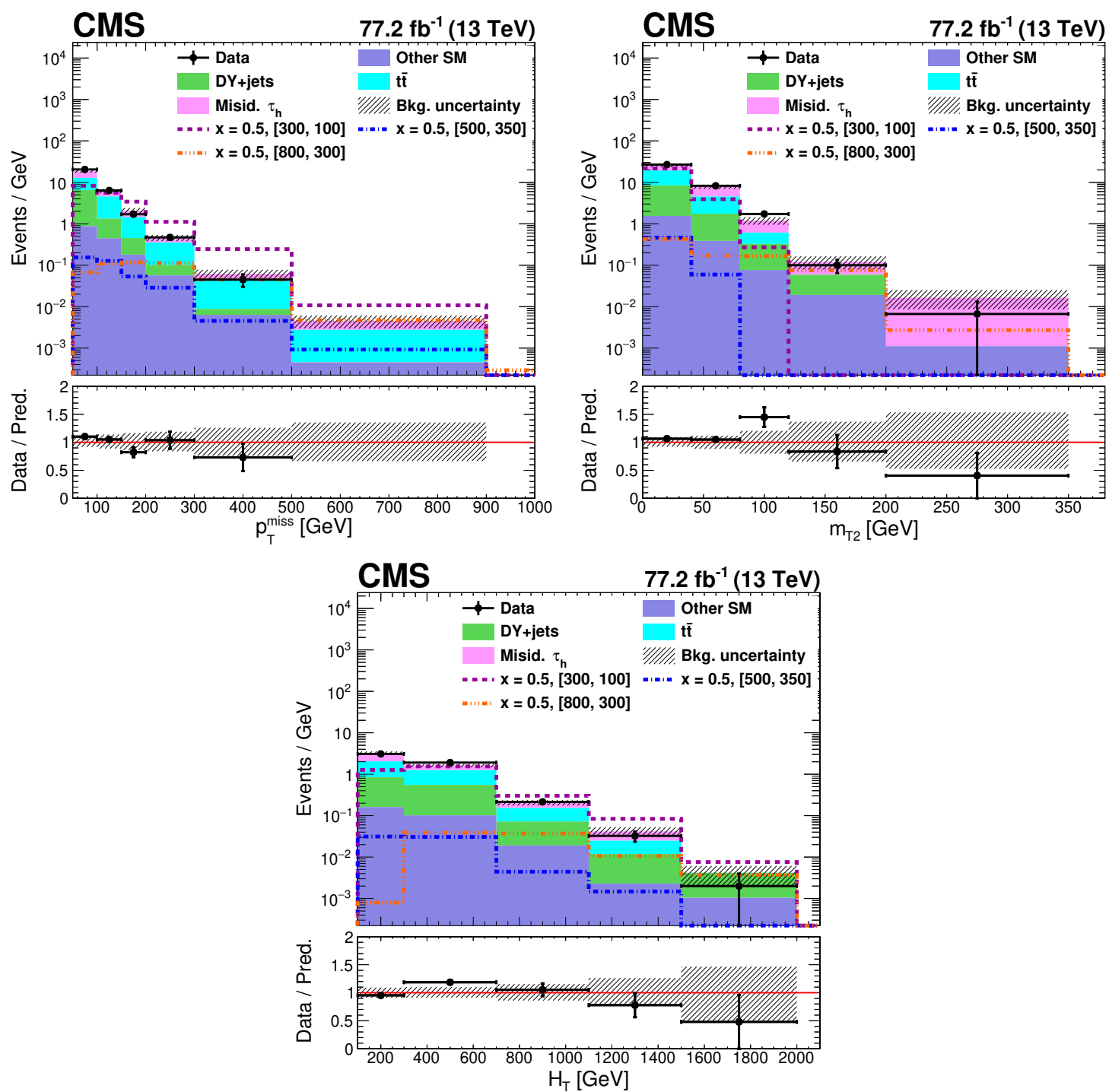

Figure 2. Distributions of the search variables $p_{\mathrm{T}}^{\text {miss }}, m_{\mathrm{T} 2}$, and $H_{\mathrm{T}}$ after event selection, for data and the predicted background. The histograms for the background processes are stacked, and the distributions for a few representative signal points corresponding to $x=0.5$ and $\left[m_{\widetilde{t}_{1}}, m_{\widetilde{\chi}_{1}^{0}}\right.$ ] $=[300,100],[500,350]$, and $[800,300] \mathrm{GeV}$ are overlaid. The lower panel indicates the ratio of the observed data to the background prediction. The shaded bands indicate the statistical and systematic uncertainties on the background, added in quadrature.

selected with e $\mu$ triggers, and are required to satisfy the same offline requirements as the $\mathrm{SR}$ with the e and $\mu$ replacing the two $\tau_{\mathrm{h}}$ candidates. The e $\mu$ triggers are $\approx 95 \%$ efficient for lepton candidates. In addition, in order to reduce possible DY contamination (from the tail of the e $\mu$ invariant mass distribution in the process $\mathrm{Z} / \gamma^{*} \rightarrow \tau \tau \rightarrow \mathrm{e} \mu$ ) in this $\mathrm{CR}$, events are vetoed if the invariant mass of the e $\mu$ system lies in the range $60<m_{\mathrm{e} \mu}<120 \mathrm{GeV}$. This selection on the dilepton invariant mass is more effective in the $\mu \mu \mathrm{CR}$ to be discussed later, but is also applied here in order to be consistent. Other objects, such as jets and 


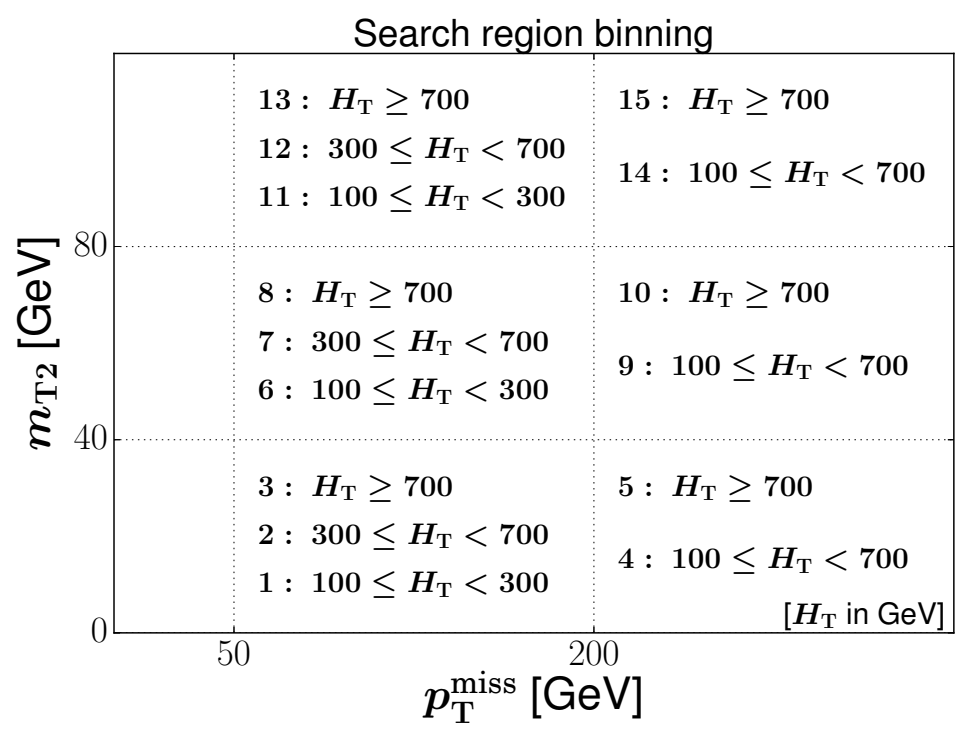

Figure 3. The 15 search regions defined in bins of $p_{\mathrm{T}}^{\text {miss }}, m_{\mathrm{T} 2}$, and $H_{\mathrm{T}}$.

b-tagged jets, are selected using the same kinematic requirements and working points as in the SR. The definitions of the search variables for this CR are the same as those in the SR, except that the e $\mu$ pair is used in place of the $\tau_{\mathrm{h}}$ pair for evaluating the search variables. The purity of $t \bar{t}$ in the $\mathrm{CR}$ (i.e., the fraction of $\mathrm{t} \overline{\mathrm{t}}$ events in each bin) is measured in simulation as $\gtrsim 85 \%$, as shown in figure 4 (upper panels).

Residual differences between data and simulation are quantified by SFs. For a given SR region $(i)$ we define

$$
S F_{i}=\frac{N_{i, \text { data }}^{\mathrm{e} \mu \mathrm{CR}}}{N_{i, \mathrm{MC}}^{\mathrm{e} \mu \mathrm{CR}}}
$$

where the numerator and the denominator represent the yields in the $\mathrm{CR}$ in data and simulation, respectively. The corrected $t \bar{t}$ yield in simulation in each region of the $\mathrm{SR}$ is then obtained as:

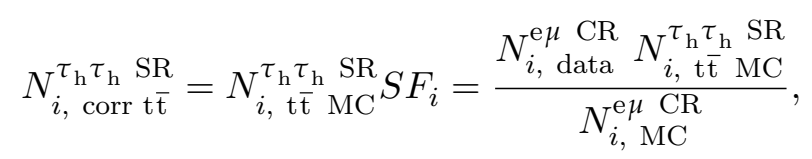

where $N_{i, \mathrm{t}}^{\tau_{\mathrm{h}} \tau_{\mathrm{h}} \text { MC }}$ is the prediction from simulated $\mathrm{t} \overline{\mathrm{t}}$ events in the SR. An alternative way of interpreting this method is that we take the $t \overline{\mathrm{t}}$ spectrum from a $\mathrm{t} \overline{\mathrm{t}}$-enriched $\mathrm{e} \mu \mathrm{CR}$ in data $\left(N_{i \text {, data }}^{\mathrm{e} \mu \mathrm{CR}}\right)$ and extrapolate it to the $\tau_{\mathrm{h}} \tau_{\mathrm{h}}$ SR by accounting for the differences between the properties of $\tau_{\mathrm{h}} \tau_{\mathrm{h}}$ and e $\mu$ final states with the ratio $N_{i, \mathrm{t} \overline{\mathrm{t}} \mathrm{MC}}^{\tau_{\mathrm{h}} \tau_{\mathrm{h}} \mathrm{SR}} / N_{i, \mathrm{MC}}^{\mathrm{e} \mu \mathrm{CR}}$ taken from simulation. The SFs in the different bins, shown in figure 4 (middle row) for both 2016 and 2017 data, are mostly found to be within $\approx 10 \%$ of unity. Note that separate SFs for bins 14 and 15 are shown for information, but these are merged and a single SF is used in subsequent calculations to reduce the statistical uncertainty.

In order to cross-check the validity of this method, the same technique is applied to an independent $\mathrm{t} \overline{\mathrm{t}}$-enriched $\mathrm{CR}$ with an oppositely charged $\mu \mu$ pair in the final state. These 

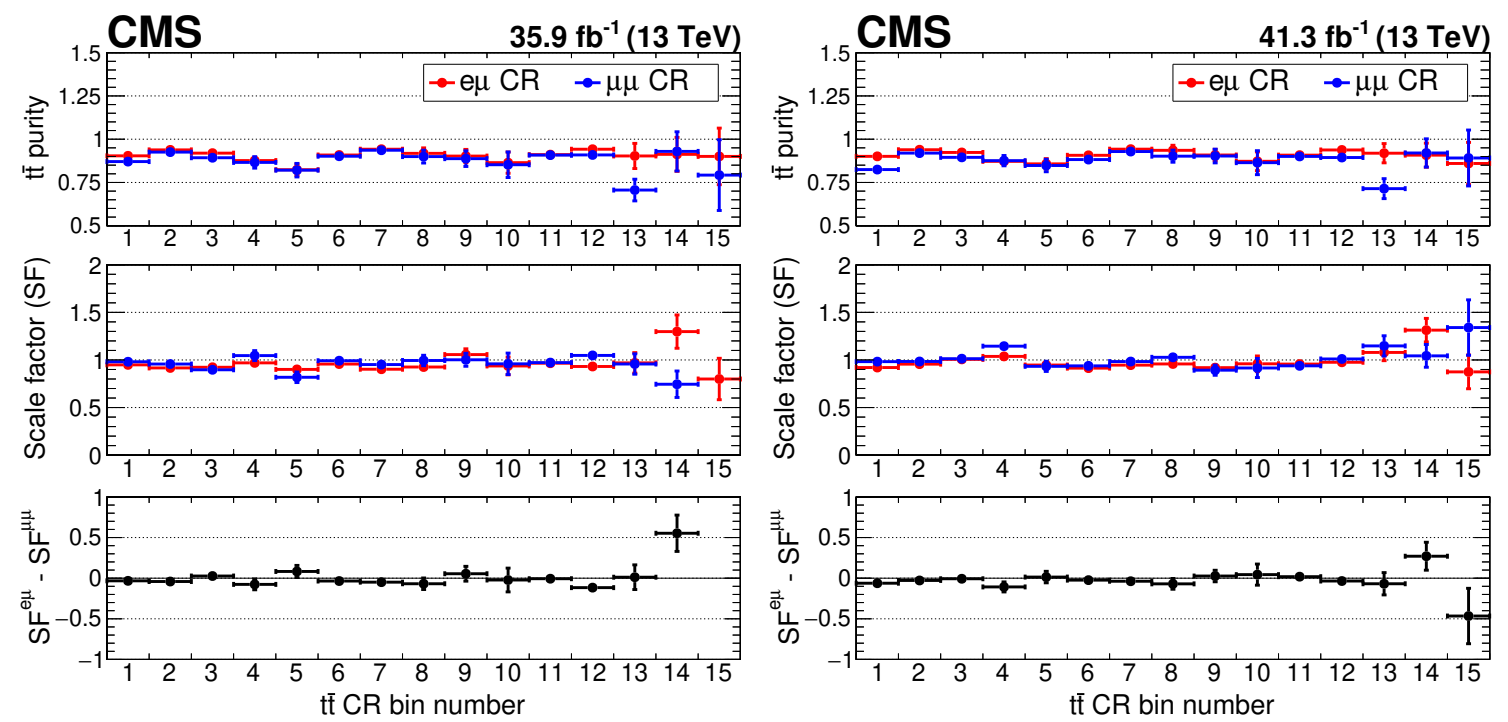

Figure 4. The purities (upper row), scale factors (middle row), and $\mathrm{SF}^{\mathrm{e} \mu}-\mathrm{SF}^{\mu \mu}$ (bottom row) in the different bins (as defined in figure 3) of the t $\overline{\mathrm{t}} \mathrm{CR}$ for 2016 (left) and 2017 (right) data. The scale factor in bin 15 of the $\mu \mu \mathrm{CR}$ in 2016 is off the visible scale. Note that bins 14 and 15 are merged to provide a single SF for subsequent calculations.

events are selected with single muon triggers that reach $\approx 95 \%$ efficiency. The event selection for the $\mu \mu \mathrm{CR}$ is similar to that for the e $\mu \mathrm{CR}$. This cross-check evaluates the effect of possible contamination from DY events (the branching fraction of $\mathrm{Z} / \gamma^{*} \rightarrow \mu \mu$ being much higher than that of $\mathrm{Z} / \gamma^{*} \rightarrow \tau \tau \rightarrow \mathrm{e} \mu$ ), and is also useful for checking any dependence of the SFs on lepton reconstruction. The differences between the SFs calculated in the main and cross-check CRs, shown in figure 4 (bottom row), are small (within $\approx 10 \%$ in most cases), and are taken as an uncertainty in the SFs. These are added in quadrature to the statistical uncertainty in the SFs, and propagated as a contribution to the uncertainty in the final $t \overline{\mathrm{t}}$ prediction.

\subsection{Misidentified hadronically decaying tau lepton candidates}

The next largest component of the total background originates from quark or gluon jets that are misidentified as a $\tau_{\mathrm{h}}$ candidate. The largest sources of such events in the SR are semileptonic and hadronic $t \overline{\mathrm{t}}$ decays. We estimate this contribution to the SR following a strategy [63] that uses the yields in $\tau_{\mathrm{h}} \tau_{\mathrm{h}} \mathrm{CRs}$, defined by inverting the requirements on the working point of the $\tau_{\mathrm{h}}$ identification.

For a genuine $\tau_{\mathrm{h}}$ passing the loose identification requirements, we define $g$ as the probability that it also passes the tight identification requirements. We define $f$ as the corresponding probability for a misidentified $\tau_{\mathrm{h}}$ candidate. We then define $N_{\mathrm{gf}}$ as the number of $\tau_{\mathrm{h}} \tau_{\mathrm{h}}$ events where the $\tau_{\mathrm{h}}$ candidate with the highest $p_{\mathrm{T}}$ is genuine and that with the second-highest $p_{\mathrm{T}}$ is misidentified, with other terms $\left(N_{\mathrm{fg}}, N_{\mathrm{gg}}\right.$, and $\left.N_{\mathrm{ff}}\right)$ defined similarly. We also define $N_{\mathrm{TL}}$ as the number of $\tau_{\mathrm{h}} \tau_{\mathrm{h}}$ events where the candidate with the highest $p_{\mathrm{T}}$ passes the tight identification criteria and that with the second-highest $p_{\mathrm{T}}$ fails, 
but passes the loose criteria, with other terms $\left(N_{\mathrm{LT}}, N_{\mathrm{LL}}\right.$, and $\left.N_{\mathrm{TT}}\right)$ defined similarly. If $N$ is the total number of events, the following set of equations can be constructed:

$$
\begin{aligned}
N & =N_{\mathrm{gg}}+N_{\mathrm{fg}}+N_{\mathrm{gf}}+N_{\mathrm{ff}}=N_{\mathrm{TT}}+N_{\mathrm{LT}}+N_{\mathrm{TL}}+N_{\mathrm{LL}}, \\
N_{\mathrm{LL}} & =\left(1-g_{1}\right)\left(1-g_{2}\right) N_{\mathrm{gg}}+\left(1-f_{1}\right)\left(1-g_{2}\right) N_{\mathrm{fg}}+\left(1-g_{1}\right)\left(1-f_{2}\right) N_{\mathrm{gf}}+\left(1-f_{1}\right)\left(1-f_{2}\right) N_{\mathrm{ff}}, \\
N_{\mathrm{LT}} & =\left(1-g_{1}\right) g_{2} N_{\mathrm{gg}}+\left(1-f_{1}\right) g_{2} N_{\mathrm{fg}}+\left(1-g_{1}\right) f_{2} N_{\mathrm{gf}}+\left(1-f_{1}\right) f_{2} N_{\mathrm{ff}}, \\
N_{\mathrm{TL}} & =g_{1}\left(1-g_{2}\right) N_{\mathrm{gg}}+f_{1}\left(1-g_{2}\right) N_{\mathrm{fg}}+g_{1}\left(1-f_{2}\right) N_{\mathrm{gf}}+f_{1}\left(1-f_{2}\right) N_{\mathrm{ff}}, \\
N_{\mathrm{TT}} & =g_{1} g_{2} N_{\mathrm{gg}}+f_{1} g_{2} N_{\mathrm{fg}}+g_{1} f_{2} N_{\mathrm{gf}}+f_{1} f_{2} N_{\mathrm{ff}},
\end{aligned}
$$

where the subscripts 1 and 2 on $g$ and $f$ refer to the $\tau_{\mathrm{h}}$ candidates with the highest and second-highest $p_{\mathrm{T}}$, respectively.

The above equations can be inverted to give the numbers of genuine and misidentified $\tau_{\mathrm{h}} \tau_{\mathrm{h}}$ candidate events in the SR:

$$
N_{\mathrm{TT}}=N_{\mathrm{TT}}^{\mathrm{gen}}+N_{\mathrm{TT}}^{\mathrm{misid}},
$$

where

$$
\begin{aligned}
N_{\mathrm{TT}}^{\mathrm{gen}} & =g_{1} g_{2} N_{\mathrm{gg}}, \\
N_{\mathrm{TT}}^{\text {misid }} & =f_{1} g_{2} N_{\mathrm{fg}}+g_{1} f_{2} N_{\mathrm{gf}}+f_{1} f_{2} N_{\mathrm{ff}} .
\end{aligned}
$$

Here $N_{\mathrm{TT}}^{\text {gen }}$ represents the number of events in the SR with two genuine $\tau_{\mathrm{h}}$ candidates in the final state, and $N_{\mathrm{TT}}^{\text {misid }}$ the number of events in the SR with one or two misidentified $\tau_{\mathrm{h}}$ candidates.

The probability $g$ is determined using t $\overline{\mathrm{t}}$ simulation, with the $\tau_{\mathrm{h}}$ candidate being matched to a generated hadronically decaying tau within a cone of radius $\Delta R=0.3$. The value of $g$ is calculated as the ratio between the number of genuine $\tau_{\mathrm{h}}$ jets passing the tight identification criteria and the number passing the loose criteria. It is evaluated as a function of the $\tau_{\mathrm{h}}$ decay modes and $p_{\mathrm{T}}$ and is observed to be about $80 \%$ with very little dependence on the $p_{\mathrm{T}}$ of the $\tau_{\mathrm{h}}$. The dependence on the decay mode is observed to be at the $10 \%$ level.

The misidentification rate $f$ is estimated using a multijet-enriched CR in data. This $\mathrm{CR}$ is defined by requiring a same-charge $\tau_{\mathrm{h}}$ pair satisfying the $\tau_{\mathrm{h}}$ selection criteria, and by requiring $p_{\mathrm{T}}^{\text {miss }}<50 \mathrm{GeV}$. The misidentification rate for a single $\tau_{\mathrm{h}}$ candidate is estimated from this CR using the following two definitions:

$$
\begin{aligned}
f(\mathrm{LL} \rightarrow \mathrm{TL}) & =\frac{\tau_{\mathrm{h}}^{1}(\mathrm{~T}) \tau_{\mathrm{h}}^{2}(\mathrm{~L})}{\tau_{\mathrm{h}}^{1}(\mathrm{~L}) \tau_{\mathrm{h}}^{2}(\mathrm{~L})}, \\
f(\mathrm{TL} \rightarrow \mathrm{TT}) & =\frac{\tau_{\mathrm{h}}^{1}(\mathrm{~T}) \tau_{\mathrm{h}}^{2}(\mathrm{~T})}{\tau_{\mathrm{h}}^{1}(\mathrm{~T}) \tau_{\mathrm{h}}^{2}(\mathrm{~L})} .
\end{aligned}
$$

Here, the term $\tau_{\mathrm{h}}^{i}(\mathrm{X})$ denotes the number of events where the candidate with the highest $(i=1)$ or second-highest $(i=2) p_{\mathrm{T}}$ passes the tight $(\mathrm{X}=\mathrm{T})$ or loose $(\mathrm{X}=\mathrm{L})$ identification criteria. In each of the two definitions above, the working point of one of the $\tau_{\mathrm{h}}$ candidates in the numerator is changed with respect to the denominator, so they could be expected 
to yield the same result. However, if the probability of one $\tau_{\mathrm{h}}$ candidate passing the tight criteria is correlated with the probability of the other to pass, differences may occur. In practice, differences of up to $\approx 10 \%$, depending on the $p_{\mathrm{T}}$ and the decay mode of the $\tau_{\mathrm{h}}$, are observed between the two definitions. These differences are used to estimate the uncertainty in this method.

The misidentification rate is measured as a function of the $\tau_{\mathrm{h}}$ decay modes and $p_{\mathrm{T}}$. It is found to be around $35 \%$ with a mild dependence on the $p_{\mathrm{T}}$ of the $\tau_{\mathrm{h}}$ candidate. The variations with decay mode are up to the $20 \%$ level. It has been found in simulation studies [63] that the misidentification rate also depends on the flavor of the parton corresponding to the jet that is misidentified as a $\tau_{\mathrm{h}}$. Since the jet flavor cannot be reliably determined in data, an additional $15 \%$ uncertainty in $f$ is included. This uncertainty is evaluated as the relative difference between the average and the maximum (or minimum) of the misidentification rates corresponding to the different jet flavors (up, down, strange, and bottom quarks, and gluons), estimated using simulated $\mathrm{W}+$ jets events.

\section{Systematic uncertainties}

There are several sources of systematic uncertainty that are propagated to the prediction of the final signal and background yields. The most significant is the uncertainty in the modeling of the identification and isolation requirements (ID-iso) [58] of the $\tau_{\mathrm{h}}$ candidates, estimated to be approximately $10 \%$ for all processes in 2016, and $20 \%$ in 2017 . The other sources of uncertainty affecting all processes include the jet energy scale (JES) and jet energy resolution (JER), the $\tau_{\mathrm{h}}$ energy scale, the effect of unclustered components in calculating $p_{\mathrm{T}}^{\text {miss }}$, pileup reweighting, and the $\mathrm{b}$ tagging efficiency. The simulation is reweighted to make its pileup distribution identical to that in data. The pileup in data depends on the measured total inelastic cross section [64], which is varied by $\pm 2.5 \%$ to obtain the uncertainty in this correction.

Since the $t \overline{\mathrm{t}}$ contribution in the SR is obtained by multiplying the simulated yield by a SF, defined as the ratio between the number of events in data and simulation, several uncertainties cancel to first order. As mentioned earlier, the difference between the t $\overline{\mathrm{t}} \mathrm{SFs}$ obtained in the $\mathrm{e} \mu$ and $\mu \mu \mathrm{CRs}$, added in quadrature with the statistical uncertainty, is taken as the uncertainty in this method. The difference between the two definitions of the misidentification rate, as defined in eq. (6.5), is taken to be the uncertainty in the misidentification rate, while the flavor dependence of the rate is accounted for by adding an additional $15 \%$ uncertainty.

The factorization $\left(\mu_{\mathrm{F}}\right)$ and renormalization $\left(\mu_{\mathrm{R}}\right)$ scales used in the simulation are varied up and down by a factor of two, avoiding the cases in which one is doubled and the other is halved. The SYSCALC package [65] has been used for this purpose. The resulting uncertainty is estimated to be less than $6 \%$ for both signal and background processes estimated from simulation. A $2.5 \%$ uncertainty in the measured integrated luminosity is used for 2016 [66], reducing to $2.3 \%$ for 2017 [67]. The uncertainty in the $\mathrm{Z}$ boson $p_{\mathrm{T}}$ correction applied to DY+jets events is taken to be equal to the deviation of the correction 
factor from unity. A normalization uncertainty of $15 \%$ is assigned to the production cross sections of the background processes that are evaluated directly from simulation [68-74].

Since the simulation of the detector for signal events is performed using FASTSim, the signal yields are corrected to account for the differences in the $\tau_{\mathrm{h}}$ identification efficiency with respect to the Geant4 simulation used for the backgrounds. The statistical uncertainty in this correction is propagated as its uncertainty. The FASTSIM package has a worse $p_{\mathrm{T}}^{\text {miss }}$ resolution than the full GEANT4 simulation, resulting in a potential artificial enhancement of the signal yields. To account for this, the signal yields are corrected, and the uncertainty in the resulting correction to the yield is estimated to be $5-10 \%$.

The uncertainties in the signal and background from all sources are presented in table 1. Upper and lower numbers correspond to the relative uncertainties due to the upward and downward variations of the respective source. These values are the weighted averages of the relative uncertainties in the different search bins with the weights being the yields in the respective bins. The tabulated sources of systematic uncertainties are modeled by lognormal distributions [75] in the likelihood function used for the statistical interpretation of the results, which is discussed in section 8. These uncertainties are considered not to be correlated with each other, but correlated across the 15 search bins. In addition, the statistical uncertainties are also taken into account and are considered to be uncorrelated across the bins.

\section{Results}

We present the observed and expected yields in all 15 search bins in table 2 along with their uncertainties. Figure 5 shows the observed data in all of the search bins, compared to the signal and background predictions.

As expected, the dominant contributions in the sensitive signal bins are from $t \bar{t}$ and misidentified $\tau_{\mathrm{h}}$ backgrounds. In cases where the background prediction of a process in a given bin is negligible, the statistical uncertainty is modeled by a gamma distribution [75] in the likelihood function used for the statistical interpretation, and the Poissonian upper limit at $68 \%$ confidence level (CL) is shown as a positive uncertainty in the table. The number of events observed in data is found to be consistent with the SM background prediction.

The test statistic used for the interpretation of the result is the profile likelihood ratio $q_{\mu}=-2 \ln \left(\mathcal{L}_{\mu} / \mathcal{L}_{\text {max }}\right)$, where $\mathcal{L}_{\mu}$ is the maximum likelihood for a fixed signal strength $\mu$, and $\mathcal{L}_{\max }$ is the global maximum of the likelihood [75]. We set upper limits on signal production at 95\% CL using a modified frequentist approach and the $\mathrm{CL}_{\mathrm{s}}$ criterion [76, 77], implemented through an asymptotic approximation of the test statistic [78]. In this calculation all the background and signal uncertainties are modeled as nuisance parameters and profiled in the maximum likelihood fit.

Final results are obtained by combining the yields from 2016 and 2017 data sets. The systematic uncertainties due to JES, factorization and renormalization scales, misidentification rate measurement, and FASTSIM $p_{\mathrm{T}}^{\text {miss }}$ correction are taken as correlated, and the rest of the uncertainties are treated as uncorrelated between the two data sets. The results are presented as observed and expected exclusion limits in the top squark and LSP mass 


\begin{tabular}{|c|c|c|c|c|c|c|c|}
\hline Uncertainty source & $\begin{array}{l}x=0.5 \\
{[300,100]}\end{array}$ & $\begin{array}{l}x=0.5 \\
{[500,350]}\end{array}$ & $\begin{array}{l}x=0.5 \\
{[800,300]}\end{array}$ & $\mathrm{t} \overline{\mathrm{t}}$ & $\mathrm{DY}+$ jets & Other SM & Misid. $\tau_{\mathrm{h}}$ \\
\hline Signal cross section & $\pm 6.7 \%$ & $\pm 7.5 \%$ & $\pm 9.5 \%$ & - & - & - & - \\
\hline FASTSIM $p_{\mathrm{T}}^{\text {miss }}$ resolution & $\pm 7.4 \%$ & $\pm 10 \%$ & $\pm 5.1 \%$ & - & - & - & - \\
\hline$\tau_{\mathrm{h}}$ FASTSIM/GEANT 4 & $\begin{array}{l}+4.4 \% \\
-4.3 \%\end{array}$ & $\begin{array}{l}+3.7 \% \\
-3.6 \%\end{array}$ & $\begin{array}{l}+7.9 \% \\
-7.5 \%\end{array}$ & - & - & - & - \\
\hline JER & $\begin{array}{l}-0.27 \% \\
<0.1 \%\end{array}$ & $\begin{array}{l}-0.81 \% \\
<0.1 \%\end{array}$ & $\begin{array}{l}<0.1 \% \\
<0.1 \%\end{array}$ & - & $\begin{array}{l}+0.47 \% \\
+0.27 \%\end{array}$ & $\begin{array}{l}-0.95 \% \\
-0.29 \%\end{array}$ & - \\
\hline JES & $\begin{array}{l}+0.18 \% \\
-0.57 \%\end{array}$ & $\begin{array}{l}<0.1 \% \\
-0.81 \%\end{array}$ & $\begin{array}{l}+0.1 \% \\
<0.1 \%\end{array}$ & - & $\begin{array}{l}+1.1 \% \\
-1.7 \%\end{array}$ & $\begin{array}{l}+0.2 \% \\
-1.6 \%\end{array}$ & - \\
\hline$\mu_{\mathrm{R}}$ and $\mu_{\mathrm{F}}$ scales & $\begin{array}{l}+0.6 \% \\
-0.7 \%\end{array}$ & $\begin{array}{l}+1.9 \% \\
-2.1 \%\end{array}$ & $\begin{array}{l}+0.31 \% \\
-0.35 \%\end{array}$ & - & $\begin{array}{l}+4.6 \% \\
-4.4 \%\end{array}$ & $\begin{array}{l}+3.2 \% \\
-2.6 \%\end{array}$ & - \\
\hline$\tau_{\mathrm{h}}$ ID-iso & $\begin{array}{l}+16 \% \\
-14 \%\end{array}$ & $\begin{array}{l}+16 \% \\
-14 \%\end{array}$ & $\begin{array}{l}+17 \% \\
-15 \%\end{array}$ & $\begin{array}{l}+16 \% \\
-15 \%\end{array}$ & $\begin{array}{l}+16 \% \\
-14 \%\end{array}$ & $\begin{array}{l}+16 \% \\
-14 \%\end{array}$ & - \\
\hline Pileup & $\begin{array}{l}<0.1 \% \\
<0.1 \%\end{array}$ & $\begin{array}{l}+0.25 \% \\
-0.18 \%\end{array}$ & $\begin{array}{l}-0.69 \% \\
+0.68 \%\end{array}$ & - & $\begin{array}{l}-0.23 \% \\
+0.24 \%\end{array}$ & $\begin{array}{l}-0.88 \% \\
+0.88 \%\end{array}$ & - \\
\hline$p_{\mathrm{T}}^{\text {miss }}$ unclustered energy & $\begin{array}{l}-0.16 \% \\
-0.78 \%\end{array}$ & $\begin{array}{l}+1 \% \\
-2 \%\end{array}$ & $\begin{array}{l}<0.1 \% \\
<0.1 \%\end{array}$ & - & $\begin{array}{l}+7 \% \\
-2 \%\end{array}$ & $\begin{array}{l}+1.3 \% \\
-1.6 \%\end{array}$ & - \\
\hline Background normalization & - & - & - & - & $\pm 15 \%$ & $\pm 15 \%$ & - \\
\hline Luminosity & $\pm 2.4 \%$ & $\pm 2.4 \%$ & $\pm 2.4 \%$ & - & $\pm 2.4 \%$ & $\pm 2.4 \%$ & - \\
\hline b tagging & $\begin{array}{l}+1.1 \% \\
-1.2 \%\end{array}$ & $\begin{array}{l}+0.65 \% \\
-0.67 \%\end{array}$ & $\begin{array}{l}+0.73 \% \\
-0.75 \%\end{array}$ & - & $\begin{array}{l}+4.7 \% \\
-4.7 \%\end{array}$ & $\begin{array}{l}+2.4 \% \\
-2.4 \%\end{array}$ & - \\
\hline$\tau_{\mathrm{h}}$ energy scale & $\begin{array}{l}+2 \% \\
-3 \%\end{array}$ & $\begin{array}{l}+2.3 \% \\
-3.3 \%\end{array}$ & $\begin{array}{l}+1.1 \% \\
-0.9 \%\end{array}$ & $\begin{array}{l}+1.5 \% \\
-1.8 \%\end{array}$ & $\begin{array}{l}+1.2 \% \\
-1.6 \%\end{array}$ & $\begin{array}{l}+0.7 \% \\
-1.3 \%\end{array}$ & - \\
\hline $\mathrm{t} \overline{\mathrm{t}} \mathrm{SF}$ & - & - & - & $\pm 2.5 \%$ & - & - & - \\
\hline $\mathrm{Z} p_{\mathrm{T}}$ reweighting & - & - & - & - & $\begin{array}{l}+9.1 \% \\
-9.1 \%\end{array}$ & - & - \\
\hline$\tau_{\mathrm{h}}$ misid. rate (parton flavour) & - & - & - & - & - & - & $\begin{array}{l}+16 \% \\
-17 \%\end{array}$ \\
\hline $\begin{array}{l}\tau_{\mathrm{h}} \text { misid. rate } \\
(\mathrm{LL} \rightarrow \mathrm{TL} \text { vs. } \mathrm{TL} \rightarrow \mathrm{TT})\end{array}$ & - & - & - & - & - & - & $\begin{array}{l}+2.7 \% \\
-2.5 \% \\
\end{array}$ \\
\hline
\end{tabular}

Table 1. Relative systematic uncertainties from different sources in signal and background yields in the 2016 and 2017 analyses combined. These values are the weighted (by the yields in the respective bins) averages of the relative uncertainties in the different search regions. For the asymmetric uncertainties, the upper (lower) entry is the uncertainty due to the upward (downward) variation, which can be in the same direction as a result of taking the weighted average. The numbers in square brackets in the heading indicate the top squark and LSP masses in GeV, respectively. 


\begin{tabular}{|lcccccc|}
\hline SR & $\mathrm{t} \overline{\mathrm{t}}$ & DY+jets & Other SM & Misid. $\tau_{\mathrm{h}}$ & Total bkg. & Data \\
\hline 1 & $170_{-8-19}^{+8+21}$ & $98_{-10-20}^{+10+22}$ & $23_{-2-4}^{+2+4}$ & $150_{-21-19}^{+21+6}$ & $441_{-25-43}^{+25+42}$ & 417 \\
2 & $200_{-8-21}^{+8+22}$ & $154_{-11-33}^{+11+35}$ & $29_{-3-5}^{+3+6}$ & $94_{-19-20}^{+19+22}$ & $477_{-23-55}^{+23+59}$ & 559 \\
3 & $20_{-3-2}^{+3+2}$ & $20_{-3-5}^{+3+6}$ & $5.1_{-0.8-1.6}^{+0.8+1.1}$ & $12_{-6-3}^{+6+4}$ & $57_{-7-8}^{+7+9}$ & 49 \\
4 & $19_{-3-2}^{+3+2}$ & $4.1_{-1.5-1.0}^{+1.5+1.0}$ & $3.8_{-0.8-0.9}^{+0.8+0.9}$ & $3.2_{-3.5-0.9}^{+3.5+1.2}$ & $30_{-5-4}^{+5+4}$ & 28 \\
5 & $8.9_{-1.6-1.0}^{+1.6+1.0}$ & $0.5_{-0.5-0.1}^{+4.8+0.1}$ & $1.6_{-0.4-0.3}^{+0.4+0.4}$ & $3.8_{-3.2-0.8}^{+3.2+1.2}$ & $15_{-4-1}^{+6+2}$ & 22 \\
6 & $51_{-4-5}^{+4+6}$ & $36_{-6-10}^{+6+9}$ & $7.8_{-1.3-1.8}^{+1.3+1.6}$ & $78_{-14-13}^{+14+12}$ & $173_{-15-20}^{+15+19}$ & 169 \\
7 & $46_{-4-5}^{+4+5}$ & $14_{-4-4}^{+4+6}$ & $5.2_{-0.8-1.1}^{+0.8+1.0}$ & $48_{-11-10}^{+11+12}$ & $113_{-12-13}^{+12+15}$ & 133 \\
8 & $4.4_{-1.3-0.5}^{+1.3+0.6}$ & $4.3_{-1.5-1.1}^{+1.5+2.0}$ & $1.4_{-0.4-0.3}^{+0.4+0.3}$ & $9.0_{-3.2-2.7}^{+3.2+3.9}$ & $19_{-4-3}^{+4+5}$ & 23 \\
9 & $3.7_{-1.1-0.4}^{+1.1+0.5}$ & $0.0_{-0.0-0.0}^{+3.5+0.0}$ & $0.7_{-0.3-0.2}^{+0.3+0.2}$ & $4.6_{-1.7-1.4}^{+1.7+2.0}$ & $9.0_{-2.0-1.5}^{+4.0+2.1}$ & 4 \\
10 & $1.0_{-0.6-0.1}^{+0.6+0.2}$ & $0.0_{-0.0-0.0}^{+3.5+0.0}$ & $0.5_{-0.2-0.1}^{+0.2+0.1}$ & $3.2_{-1.4-1.0}^{+1.4+1.3}$ & $4.7_{-1.6-1.0}^{+3.8+1.3}$ & 1 \\
11 & $6.8_{-1.6-0.9}^{+1.6+1.2}$ & $2.4_{-1.5-0.7}^{+1.5+1.3}$ & $1.4_{-0.4-0.3}^{+0.4+0.7}$ & $16_{-6-3}^{+6+4}$ & $27_{-6-4}^{+6+5}$ & 30 \\
12 & $2.9_{-1.0-0.3}^{+1.0+0.4}$ & $8.3_{-2.5-1.8}^{+2.5+2.8}$ & $2.1_{-0.3-0.4}^{+0.3+0.4}$ & $11_{-6-2}^{+6+2}$ & $24_{-7-3}^{+7+4}$ & 41 \\
13 & $0.7_{-0.5-0.4}^{+0.9+0.2}$ & $2.2_{-0.9-0.9}^{+0.9+0.6}$ & $0.7_{-0.2-0.2}^{+0.2+0.2}$ & $3.5_{-2.1-0.9}^{+2.1+1.3}$ & $7.1_{-2.3-1.4}^{+2.4+1.5}$ & 6 \\
14 & $0.5_{-0.5-0.1}^{+0.9+0.1}$ & $0.0_{-0.0-0.0}^{+3.5+0.0}$ & $0.6_{-0.2-0.1}^{+0.2+0.1}$ & $0.0_{-0.0-0.0}^{+1.8+0.0}$ & $1.1_{-0.6-0.2}^{+4.1+0.2}$ & 1 \\
15 & $0.3_{-0.3-0.1}^{+0.8+0.1}$ & $0.4_{-0.4-0.1}^{+2.2+0.1}$ & $0.1_{-0.0-0.0}^{+0.0+0.0}$ & $1.0_{-0.0-0.3}^{+1.8+0.4}$ & $1.8_{-0.5-0.3}^{+3.0+0.5}$ & 0 \\
Total & $535_{-14-58}^{+14+63}$ & $344_{-17-73}^{+19+78}$ & $83_{-4-16}^{+4+16}$ & $437_{-35-78}^{+35+73}$ & $1400_{-42-123}^{+43+125}$ & 1483 \\
\hline
\end{tabular}

Table 2. Event yields along with statistical and systematic uncertainties in the 2016 and 2017 analyses combined, for different background sources and the total background in the 15 search bins, as defined in figure 3. The uncertainties that are smaller than 0.05 are listed as 0.0 . The number of events observed in data is also shown. The notation used is yield -stat-syst $_{\text {stat }}$.

plane in figure 6. Top squark masses up to $1100 \mathrm{GeV}$ are excluded for a nearly massless LSP, and LSP masses up to $450 \mathrm{GeV}$ are excluded for a top squark mass of $900 \mathrm{GeV}$. The exclusion limits are not very sensitive to the choice of $x$ because of the complementary nature of the signal diagrams, as discussed in section 3 .

The most sensitive search bins for the higher top squark masses are 14 and 15 . The observed data in these two bins are lower than the total background prediction, resulting in the observed limit being higher than the expected one. Hence, even though there are more events in data than prediction overall, the observed mass limit is stronger than expected. The excesses are primarily in bins $2,5,7$, and 12 which are more significant for low top squark masses, hence the observed limit is slightly worse than expected in that region. The limits become weaker with decreasing $\Delta m=m_{\widetilde{\mathrm{t}}_{1}}-m_{\widetilde{\chi}_{1}^{0}}$, corresponding to a parameter space with final-state particles having lower momentum and hence less sensitivity. 


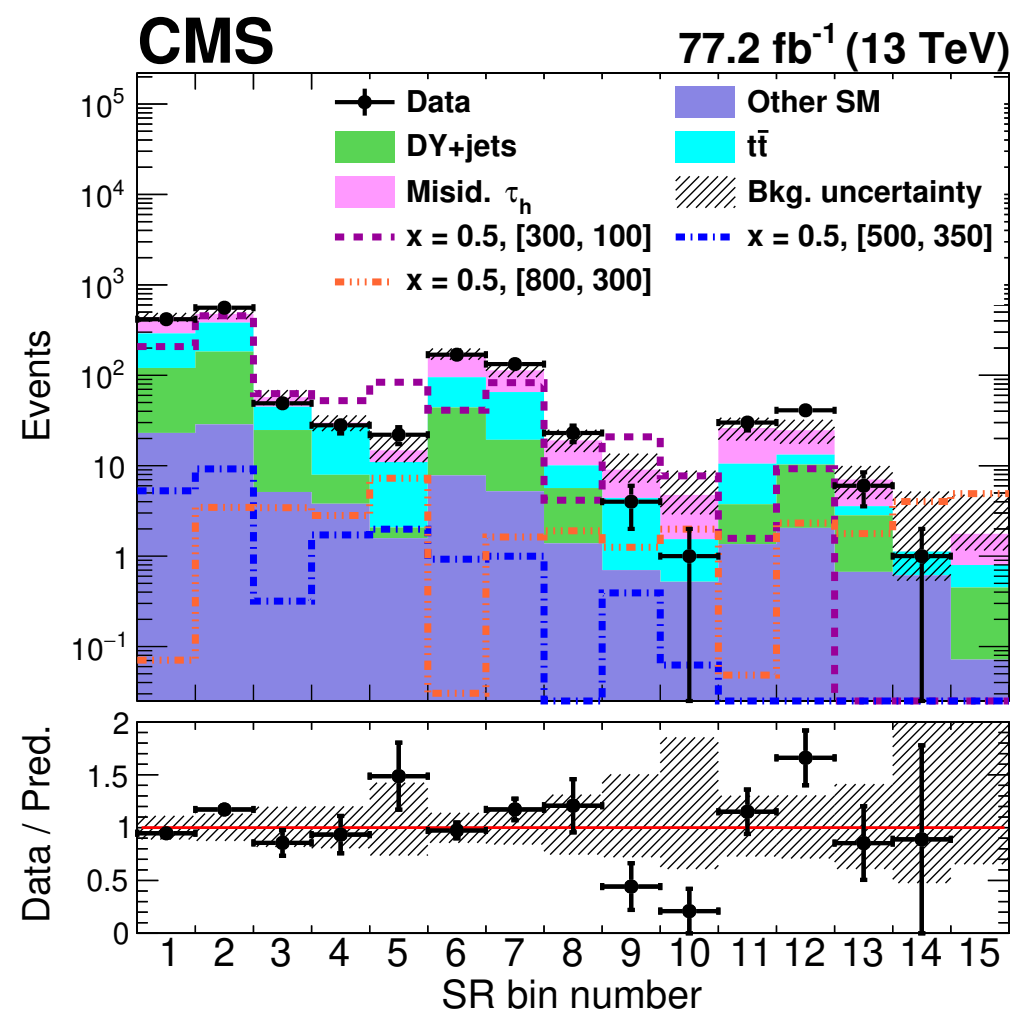

Figure 5. Event yields in the 15 search bins as defined in figure 3. The yields for the background processes are stacked, and those for a few representative signal points corresponding to $x=0.5$ and $\left[m_{\widetilde{\mathfrak{t}}_{1}}, m_{\widetilde{\chi}_{1}}\right]=[300,100],[500,350]$, and $[800,300] \mathrm{GeV}$ are overlaid. The lower panel indicates the ratio of the observed data to the background prediction in each bin. The shaded bands indicate the statistical and systematic uncertainties in the background, added in quadrature.

\section{Summary}

The signature of top squark pair production in final states with two tau leptons has been explored in data collected with the CMS detector during 2016 and 2017, corresponding to integrated luminosities of 35.9 and $41.3 \mathrm{fb}^{-1}$, respectively. The search was performed in the final state containing an oppositely charged hadronic tau lepton pair, at least one jet identified as likely to originate from the fragmentation of a b quark, and missing transverse momentum. The dominant standard model backgrounds were found to originate from top quark pair production and processes where jets were misidentified as hadronic tau lepton decays. Control samples in data were used to estimate these backgrounds, while other backgrounds were estimated using simulation.

No significant excess was observed, and exclusion limits on the top squark mass in terms of the mass of the lightest neutralino were set at $95 \%$ confidence level within the framework of simplified models where the top squark decays via a chargino to final states including tau leptons. In such models, top squark masses are excluded up to $1100 \mathrm{GeV}$ for an almost massless neutralino, and LSP masses up to $450 \mathrm{GeV}$ are excluded for a top squark mass of $900 \mathrm{GeV}$. These results probe a region of the supersymmetric parameter space corresponding to high-tan $\beta$ and higgsino-like scenarios. 

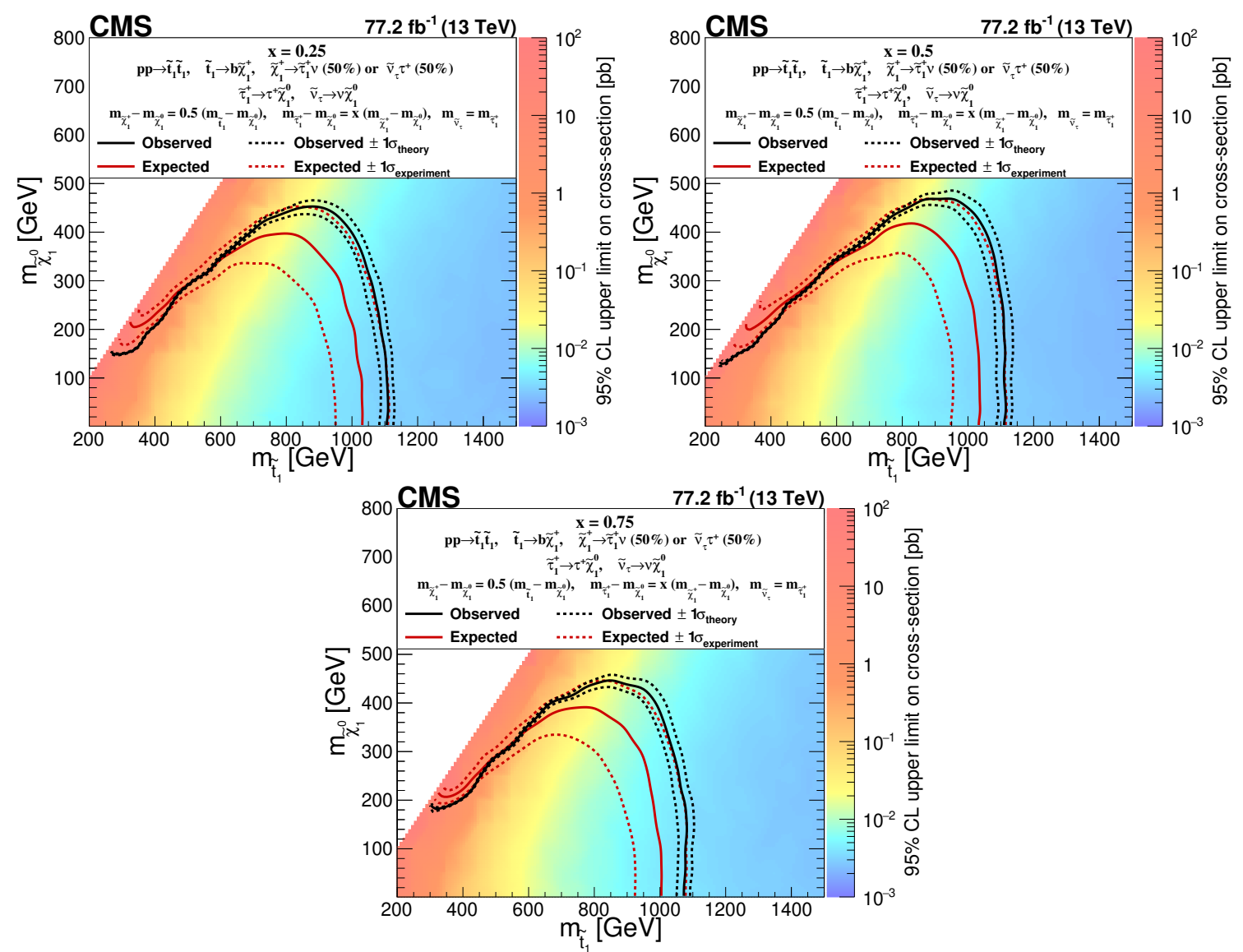

Figure 6. Exclusion limits at $95 \%$ CL for the pair production of top squarks decaying to a $\tau_{\mathrm{h}} \tau_{\mathrm{h}}$ final state, displayed in the $m_{\widetilde{\mathrm{t}}_{1}}-m_{\widetilde{\chi}_{1}^{0}}$ plane for $x=0.25$ (upper left), 0.5 (upper right) and 0.75 (lower), as described in eq. (3.1). The color axis represents the observed limit in the cross section, while the black (red) lines represent the observed (expected) mass limits. The signal cross sections are evaluated using NNLO plus next-to-leading logarithmic (NLL) calculations. The solid lines represent the central values. The dashed red lines indicate the region containing $68 \%$ of the distribution of limits expected under the background-only hypothesis. The dashed black lines show the change in the observed limit due to variation of the signal cross sections within their theoretical uncertainties.

\section{Acknowledgments}

We congratulate our colleagues in the CERN accelerator departments for the excellent performance of the LHC and thank the technical and administrative staffs at CERN and at other CMS institutes for their contributions to the success of the CMS effort. In addition, we gratefully acknowledge the computing centers and personnel of the Worldwide LHC Computing Grid for delivering so effectively the computing infrastructure essential to our analyses. Finally, we acknowledge the enduring support for the construction and operation of the LHC and the CMS detector provided by the following funding agencies: BMBWF and FWF (Austria); FNRS and FWO (Belgium); CNPq, CAPES, FAPERJ, FAPERGS, and FAPESP (Brazil); MES (Bulgaria); CERN; CAS, MoST, and NSFC (China); COL- 
CIENCIAS (Colombia); MSES and CSF (Croatia); RPF (Cyprus); SENESCYT (Ecuador); MoER, ERC IUT, PUT and ERDF (Estonia); Academy of Finland, MEC, and HIP (Finland); CEA and CNRS/IN2P3 (France); BMBF, DFG, and HGF (Germany); GSRT (Greece); NKFIA (Hungary); DAE and DST (India); IPM (Iran); SFI (Ireland); INFN (Italy); MSIP and NRF (Republic of Korea); MES (Latvia); LAS (Lithuania); MOE and UM (Malaysia); BUAP, CINVESTAV, CONACYT, LNS, SEP, and UASLP-FAI (Mexico); MOS (Montenegro); MBIE (New Zealand); PAEC (Pakistan); MSHE and NSC (Poland); FCT (Portugal); JINR (Dubna); MON, RosAtom, RAS, RFBR, and NRC KI (Russia); MESTD (Serbia); SEIDI, CPAN, PCTI, and FEDER (Spain); MOSTR (Sri Lanka); Swiss Funding Agencies (Switzerland); MST (Taipei); ThEPCenter, IPST, STAR, and NSTDA (Thailand); TUBITAK and TAEK (Turkey); NASU (Ukraine); STFC (United Kingdom); DOE and NSF (U.S.A.).

Individuals have received support from the Marie-Curie program and the European Research Council and Horizon 2020 Grant, contract Nos. 675440, 752730, and 765710 (European Union); the Leventis Foundation; the A.P. Sloan Foundation; the Alexander von Humboldt Foundation; the Belgian Federal Science Policy Office; the Fonds pour la Formation à la Recherche dans l'Industrie et dans l'Agriculture (FRIA-Belgium); the Agentschap voor Innovatie door Wetenschap en Technologie (IWT-Belgium); the F.R.S.-FNRS and FWO (Belgium) under the "Excellence of Science - EOS" — be.h project n. 30820817; the Beijing Municipal Science \& Technology Commission, No. Z181100004218003; the Ministry of Education, Youth and Sports (MEYS) of the Czech Republic; the Lendület ("Momentum") Program and the János Bolyai Research Scholarship of the Hungarian Academy of Sciences, the New National Excellence Program ÚNKP, the NKFIA research grants 123842, 123959, 124845, 124850, 125105, 128713, 128786, and 129058 (Hungary); the Council of Science and Industrial Research, India; the HOMING PLUS program of the Foundation for Polish Science, cofinanced from European Union, Regional Development Fund, the Mobility Plus program of the Ministry of Science and Higher Education, the National Science Center (Poland), contracts Harmonia 2014/14/M/ST2/00428, Opus 2014/13/B/ST2/02543, 2014/15/B/ST2/03998, and 2015/19/B/ST2/02861, Sonatabis 2012/07/E/ST2/01406; the National Priorities Research Program by Qatar National Research Fund; the Ministry of Science and Education, grant no. 3.2989.2017 (Russia); the Programa Estatal de Fomento de la Investigación Científica y Técnica de Excelencia María de Maeztu, grant MDM-2015-0509 and the Programa Severo Ochoa del Principado de Asturias; the Thalis and Aristeia programs cofinanced by EU-ESF and the Greek NSRF; the Rachadapisek Sompot Fund for Postdoctoral Fellowship, Chulalongkorn University and the Chulalongkorn Academic into Its 2nd Century Project Advancement Project (Thailand); the Nvidia Corporation; the Welch Foundation, contract C-1845; and the Weston Havens Foundation (U.S.A.).

Open Access. This article is distributed under the terms of the Creative Commons Attribution License (CC-BY 4.0), which permits any use, distribution and reproduction in any medium, provided the original author(s) and source are credited. 


\section{References}

[1] P. Ramond, Dual Theory for Free Fermions, Phys. Rev. D 3 (1971) 2415 [InSPIRE].

[2] Yu.A. Golfand and E.P. Likhtman, Extension of the Algebra of Poincaré Group Generators and Violation of $p$ Invariance, JETP Lett. 13 (1971) 323 [INSPIRE].

[3] A. Neveu and J.H. Schwarz, Factorizable dual model of pions, Nucl. Phys. B 31 (1971) 86 [INSPIRE].

[4] J. Wess and B. Zumino, A Lagrangian model invariant under supergauge transformations, Phys. Lett. 49B (1974) 52 [inSPIRE].

[5] P. Fayet, Supergauge invariant extension of the Higgs mechanism and a model for the electron and its neutrino, Nucl. Phys. B 90 (1975) 104 [INSPIRE].

[6] G. 't Hooft, Naturalness, chiral symmetry and spontaneous chiral symmetry breaking, NATO Sci. Ser. B 59 (1980) 135 [inSPIRE].

[7] R.K. Kaul and P. Majumdar, Cancellation of quadratically divergent mass corrections in globally supersymmetric spontaneously broken gauge theories, Nucl. Phys. B 199 (1982) 36 [INSPIRE].

[8] H.P. Nilles, Supersymmetry, supergravity and particle physics, Phys. Rept. 110 (1984) 1 [INSPIRE].

[9] S.P. Martin, A supersymmetry primer, hep-ph/9709356 [INSPIRE].

[10] G.R. Farrar and P. Fayet, Phenomenology of the production, decay and detection of new hadronic states associated with supersymmetry, Phys. Lett. 76B (1978) 575 [INSPIRE].

[11] H. Baer, C.-h. Chen, M. Drees, F. Paige and X. Tata, Collider phenomenology for supersymmetry with large tan $\beta$, Phys. Rev. Lett. 79 (1997) 986 [Erratum ibid. 80 (1998) 642] [hep-ph/9704457] [INSPIRE].

[12] M. Guchait and D.P. Roy, Using $\tau$ polarization as a distinctive SUGRA signature at LHC, Phys. Lett. B 541 (2002) 356 [hep-ph/0205015] [INSPIRE].

[13] J. Alwall, P. Schuster and N. Toro, Simplified models for a first characterization of new physics at the LHC, Phys. Rev. D 79 (2009) 075020 [arXiv:0810.3921] [InSPIRE].

[14] LHC New Physics Working Group collaboration, Simplified models for LHC new physics searches, J. Phys. G 39 (2012) 105005 [arXiv:1105.2838] [InSPIRE].

[15] CMS collaboration, Search for top squark pair production in pp collisions at $\sqrt{s}=13$ TeV using single lepton events, JHEP 10 (2017) 019 [arXiv:1706.04402] [INSPIRE].

[16] CMS collaboration, Search for top squarks and dark matter particles in opposite-charge dilepton final states at $\sqrt{s}=13$ TeV, Phys. Rev. D 97 (2018) 032009 [arXiv: 1711.00752] [INSPIRE].

[17] CMS collaboration, Search for top-squark pair production in the single-lepton final state in pp collisions at $\sqrt{s}=8 \mathrm{TeV}$, Eur. Phys. J. C 73 (2013) 2677 [arXiv:1308.1586] [InSPIRE].

[18] CMS collaboration, Search for direct pair production of scalar top quarks in the single-and dilepton channels in proton-proton collisions at $\sqrt{s}=8$ TeV, JHEP 07 (2016) 027 [Erratum ibid. 09 (2016) 056] [arXiv:1602.03169] [INSPIRE].

[19] CMS collaboration, Search for top squark pair production in compressed-mass-spectrum scenarios in proton-proton collisions at $\sqrt{s}=8$ TeV using the $\alpha_{T}$ variable, Phys. Lett. B 767 (2017) 403 [arXiv: 1605.08993] [INSPIRE]. 
[20] CMS collaboration, Searches for pair production of third-generation squarks in $\sqrt{s}=13$ TeV pp collisions, Eur. Phys. J. C 77 (2017) 327 [arXiv:1612.03877] [InSPIRE].

[21] CMS collaboration, Search for direct production of supersymmetric partners of the top quark in the all-jets final state in proton-proton collisions at $\sqrt{s}=13 \mathrm{TeV}$, JHEP 10 (2017) 005 [arXiv: 1707.03316] [INSPIRE].

[22] CMS collaboration, Search for supersymmetry in proton-proton collisions at 13 TeV using identified top quarks, Phys. Rev. D 97 (2018) 012007 [arXiv:1710.11188] [INSPIRE].

[23] ATLAS collaboration, Search for direct top squark pair production in final states with two leptons in $\sqrt{s}=13$ TeV pp collisions with the ATLAS detector, Eur. Phys. J. C 77 (2017) 898 [arXiv: 1708.03247] [INSPIRE].

[24] ATLAS collaboration, ATLAS Run 1 searches for direct pair production of third-generation squarks at the Large Hadron Collider, Eur. Phys. J. C 75 (2015) 510 [Erratum ibid. C 76 (2016) 153] [arXiv:1506.08616] [INSPIRE].

[25] ATLAS collaboration, Search for top squark pair production in final states with one isolated lepton, jets and missing transverse momentum in $\sqrt{s}=8 \mathrm{TeV}$ pp collisions with the ATLAS detector, JHEP 11 (2014) 118 [arXiv:1407.0583] [INSPIRE].

[26] ATLAS collaboration, Search for direct top-squark pair production in final states with two leptons in pp collisions at $\sqrt{s}=8$ TeV with the ATLAS detector, JHEP 06 (2014) 124 [arXiv:1403.4853] [INSPIRE].

[27] ATLAS collaboration, Search for top squarks in final states with one isolated lepton, jets and missing transverse momentum in $\sqrt{s}=13$ TeV pp collisions with the ATLAS detector, Phys. Rev. D 94 (2016) 052009 [arXiv: 1606.03903] [INSPIRE].

[28] ATLAS collaboration, Search for top squarks decaying to tau sleptons in pp collisions at $\sqrt{s}=13$ TeV with the ATLAS detector, Phys. Rev. D 98 (2018) 032008 [arXiv:1803.10178] [INSPIRE].

[29] CMS collaboration, The CMS experiment at the CERN LHC, 2008 JINST 3 S08004 [INSPIRE].

[30] CMS collaboration, The CMS trigger system, 2017 JINST 12 P01020 [arXiv:1609.02366] [INSPIRE].

[31] C. Oleari, The Powheg BOX, Nucl. Phys. Proc. Suppl. B 205-206 (2010) 36 [inSPIRE].

[32] P. Nason, A new method for combining NLO QCD with shower Monte Carlo algorithms, JHEP 11 (2004) 040 [hep-ph/0409146] [INSPIRE].

[33] S. Frixione, P. Nason and C. Oleari, Matching NLO QCD computations with parton shower simulations: the POWHEG method, JHEP 11 (2007) 070 [arXiv:0709.2092] [INSPIRE].

[34] S. Alioli, P. Nason, C. Oleari and E. Re, A general framework for implementing NLO calculations in shower Monte Carlo programs: the POWHEG BOX, JHEP 06 (2010) 043 [arXiv: 1002.2581] [INSPIRE].

[35] S. Frixione, P. Nason and G. Ridolfi, A positive-weight next-to-leading-order Monte Carlo for heavy flavour hadroproduction, JHEP 09 (2007) 126 [arXiv:0707.3088] [INSPIRE].

[36] S. Alioli, P. Nason, C. Oleari and E. Re, NLO single-top production matched with shower in POWHEG: s- and t-channel contributions, JHEP 09 (2009) 111 [Erratum ibid. 02 (2010) 011] [arXiv:0907.4076] [INSPIRE]. 
[37] J. Alwall et al., The automated computation of tree-level and next-to-leading order differential cross sections and their matching to parton shower simulations, JHEP 07 (2014) 079 [arXiv: 1405.0301] [INSPIRE].

[38] T. Sjöstrand et al., An Introduction to PYTHIA 8.2, Comput. Phys. Commun. 191 (2015) 159 [arXiv: 1410.3012] [INSPIRE].

[39] CMS collaboration, Investigations of the impact of the parton shower tuning in PYTHIA 8 in the modelling of $\mathrm{t} \overline{\mathrm{t}}$ at $\sqrt{s}=8$ and $13 \mathrm{TeV}$, CMS-PAS-TOP-16-021 (2016).

[40] CMS collaboration, Event generator tunes obtained from underlying event and multiparton scattering measurements, Eur. Phys. J. C 76 (2016) 155 [arXiv:1512.00815] [INSPIRE].

[41] CMS collaboration, Extraction and validation of a new set of CMS PYTHIA8 tunes from underlying-event measurements, Eur. Phys. J. C 80 (2020) 4 [arXiv:1903.12179] [INSPIRE].

[42] GEANT4 collaboration, Geant4 - a simulation toolkit, Nucl. Instrum. Meth. A 506 (2003) 250 [INSPIRE].

[43] W. Beenakker, R. Hopker, M. Spira and P.M. Zerwas, Squark and gluino production at hadron colliders, Nucl. Phys. B 492 (1997) 51 [hep-ph/9610490] [INSPIRE].

[44] A. Kulesza and L. Motyka, Threshold resummation for squark-antisquark and gluino-pair production at the LHC, Phys. Rev. Lett. 102 (2009) 111802 [arXiv:0807.2405] [INSPIRE].

[45] A. Kulesza and L. Motyka, Soft gluon resummation for the production of gluino-gluino and squark-antisquark pairs at the LHC, Phys. Rev. D 80 (2009) 095004 [arXiv:0905.4749] [INSPIRE].

[46] W. Beenakker, S. Brensing, M. Krämer, A. Kulesza, E. Laenen and I. Niessen, Soft-gluon resummation for squark and gluino hadroproduction, JHEP 12 (2009) 041 [arXiv: 0909.4418] [INSPIRE].

[47] W. Beenakker et al., Squark and gluino hadroproduction, Int. J. Mod. Phys. A 26 (2011) 2637 [arXiv: 1105.1110] [INSPIRE].

[48] A. Giammanco, The Fast Simulation of the CMS Experiment, J. Phys. Conf. Ser. 513 (2014) 022012 [INSPIRE].

[49] CMS collaboration, Particle-flow reconstruction and global event description with the CMS detector, 2017 JINST 12 P10003 [arXiv:1706.04965] [INSPIRE].

[50] M. Cacciari, G.P. Salam and G. Soyez, The anti- $k_{t}$ jet clustering algorithm, JHEP 04 (2008) 063 [arXiv: 0802.1189] [INSPIRE].

[51] M. Cacciari, G.P. Salam and G. Soyez, FastJet user manual, Eur. Phys. J. C 72 (2012) 1896 [arXiv: 1111.6097] [INSPIRE].

[52] CMS collaboration, Jet energy scale and resolution in the CMS experiment in pp collisions at $8 \mathrm{TeV}, 2017$ JINST $12 \mathrm{P} 02014$ [arXiv:1607.03663] [INSPIRE].

[53] CMS collaboration, Jet algorithms performance in 13 TeV data, CMS-PAS-JME-16-003 (2017).

[54] CMS collaboration, Identification of heavy-flavour jets with the CMS detector in pp collisions at $13 \mathrm{TeV}, 2018$ JINST $13 \mathrm{P} 05011$ [arXiv: 1712.07158] [INSPIRE].

[55] CMS collaboration, Performance of electron reconstruction and selection with the CMS detector in proton-proton collisions at $\sqrt{s}=8 \mathrm{TeV}, 2015$ JINST $10 \mathrm{P} 06005$ [arXiv: 1502.02701] [INSPIRE]. 
[56] CMS collaboration, Performance of the CMS muon detector and muon reconstruction with proton-proton collisions at $\sqrt{s}=13$ TeV, 2018 JINST 13 P06015 [arXiv:1804.04528] [INSPIRE].

[57] CMS collaboration, Performance of missing transverse momentum reconstruction in proton-proton collisions at $\sqrt{s}=13$ TeV using the CMS detector, 2019 JINST 14 P07004 [arXiv: 1903.06078] [INSPIRE].

[58] CMS collaboration, Performance of reconstruction and identification of $\tau$ leptons decaying to hadrons and $\nu_{\tau}$ in pp collisions at $\sqrt{s}=13$ TeV, 2018 JINST 13 P10005 [arXiv: 1809.02816] [INSPIRE].

[59] C.G. Lester and D.J. Summers, Measuring masses of semiinvisibly decaying particles pair produced at hadron colliders, Phys. Lett. B 463 (1999) 99 [hep-ph/9906349] [INSPIRE].

[60] A. Barr, C. Lester and P. Stephens, $m_{\mathrm{T} 2}$ : The Truth behind the glamour, J. Phys. G 29 (2003) 2343 [hep-ph/0304226] [INSPIRE].

[61] A.J. Barr and C. Gwenlan, The Race for supersymmetry: Using m(T2) for discovery, Phys. Rev. D 80 (2009) 074007 [arXiv:0907.2713] [INSPIRE].

[62] CMS collaboration, Search for heavy neutrinos and third-generation leptoquarks in hadronic states of two $\tau$ leptons and two jets in proton-proton collisions at $\sqrt{s}=13$ TeV, JHEP 03 (2019) 170 [arXiv:1811.00806] [INSPIRE].

[63] CMS collaboration, Search for supersymmetry in events with a $\tau$ lepton pair and missing transverse momentum in proton-proton collisions at $\sqrt{s}=13 \mathrm{TeV}$, JHEP 11 (2018) 151 [arXiv: 1807.02048] [INSPIRE].

[64] CMS collaboration, Measurement of the inelastic proton-proton cross section at $\sqrt{s}=13 \mathrm{TeV}$, JHEP 07 (2018) 161 [arXiv: 1802.02613] [INSPIRE].

[65] A. Kalogeropoulos and J. Alwall, The SysCalc code: A tool to derive theoretical systematic uncertainties, arXiv: 1801.08401 [INSPIRE].

[66] CMS collaboration, CMS luminosity measurements for the 2016 data taking period, CMS-PAS-LUM-17-001 (2017).

[67] CMS collaboration, CMS luminosity measurement for the 2017 data-taking period at $\sqrt{s}=13$ TeV, CMS-PAS-LUM-17-004 (2018).

[68] CMS collaboration, Measurement of the differential Drell-Yan cross section in proton-proton collisions at $\sqrt{\mathrm{s}}=13$ TeV, JHEP 12 (2019) 059 [arXiv: 1812.10529] [INSPIRE].

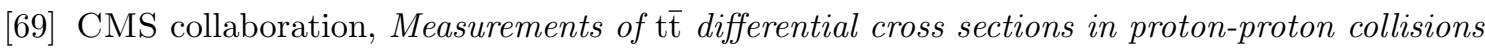
at $\sqrt{s}=13$ TeV using events containing two leptons, JHEP 02 (2019) 149 [arXiv: 1811.06625] [INSPIRE].

[70] ATLAS collaboration, Measurement of the $W^{+} W^{-}$production cross section in pp collisions at a centre-of-mass energy of $\sqrt{s}=13$ TeV with the ATLAS experiment, Phys. Lett. B 773 (2017) 354 [arXiv:1702.04519] [INSPIRE].

[71] CMS collaboration, Measurement of top quark pair production in association with a $Z$ boson in proton-proton collisions at $\sqrt{s}=13 \mathrm{TeV}$, submitted to JHEP [arXiv:1907.11270] [INSPIRE].

[72] CMS collaboration, Measurement of differential cross sections and charge ratios for t-channel single top quark production in proton-proton collisions at $\sqrt{s}=13 \mathrm{Te} V$, submitted to Eur. Phys. J. C [arXiv: 1907.08330] [INSPIRE]. 
[73] CMS collaboration, Measurements of the $p p \rightarrow W Z$ inclusive and differential production cross section and constraints on charged anomalous triple gauge couplings at $\sqrt{s}=13$ TeV, JHEP 04 (2019) 122 [arXiv: 1901.03428] [INSPIRE].

[74] CMS collaboration, Measurement of the differential cross sections for the associated production of a $W$ boson and jets in proton-proton collisions at $\sqrt{s}=13$ TeV, Phys. Rev. D 96 (2017) 072005 [arXiv: 1707.05979] [INSPIRE].

[75] ATLAS and CMS collaborations and the LHC Higgs Combination Group, Procedure for the LHC Higgs boson search combination in Summer 2011, CMS-NOTE-2011-005 (2011).

[76] T. Junk, Confidence level computation for combining searches with small statistics, Nucl. Instrum. Meth. A 434 (1999) 435 [hep-ex/9902006] [InSPIRE].

[77] A.L. Read, Presentation of search results: the CL $L_{s}$ technique, J. Phys. G 28 (2002) 2693 [INSPIRE].

[78] G. Cowan, K. Cranmer, E. Gross and O. Vitells, Asymptotic formulae for likelihood-based tests of new physics, Eur. Phys. J. C 71 (2011) 1554 [Erratum ibid. C 73 (2013) 2501] [arXiv:1007.1727] [INSPIRE]. 


\section{The CMS collaboration}

Yerevan Physics Institute, Yerevan, Armenia

A.M. Sirunyan ${ }^{\dagger}$, A. Tumasyan

\section{Institut für Hochenergiephysik, Wien, Austria}

W. Adam, F. Ambrogi, T. Bergauer, J. Brandstetter, M. Dragicevic, J. Erö, A. Escalante Del Valle, M. Flechl, R. Frühwirth ${ }^{1}$, M. Jeitler ${ }^{1}$, N. Krammer, I. Krätschmer, D. Liko, T. Madlener, I. Mikulec, N. Rad, J. Schieck ${ }^{1}$, R. Schöfbeck, M. Spanring, D. Spitzbart, W. Waltenberger, C.-E. Wulz ${ }^{1}$, M. Zarucki

Institute for Nuclear Problems, Minsk, Belarus

V. Drugakov, V. Mossolov, J. Suarez Gonzalez

\section{Universiteit Antwerpen, Antwerpen, Belgium}

M.R. Darwish, E.A. De Wolf, D. Di Croce, X. Janssen, A. Lelek, M. Pieters, H. Rejeb Sfar, H. Van Haevermaet, P. Van Mechelen, S. Van Putte, N. Van Remortel

\section{Vrije Universiteit Brussel, Brussel, Belgium}

F. Blekman, E.S. Bols, S.S. Chhibra, J. D'Hondt, J. De Clercq, D. Lontkovskyi, S. Lowette, I. Marchesini, S. Moortgat, Q. Python, K. Skovpen, S. Tavernier, W. Van Doninck, P. Van Mulders

\section{Université Libre de Bruxelles, Bruxelles, Belgium}

D. Beghin, B. Bilin, H. Brun, B. Clerbaux, G. De Lentdecker, H. Delannoy, B. Dorney, L. Favart, A. Grebenyuk, A.K. Kalsi, A. Popov, N. Postiau, E. Starling, L. Thomas, C. Vander Velde, P. Vanlaer, D. Vannerom

\section{Ghent University, Ghent, Belgium}

T. Cornelis, D. Dobur, I. Khvastunov ${ }^{2}$, M. Niedziela, C. Roskas, D. Trocino, M. Tytgat, W. Verbeke, B. Vermassen, M. Vit, N. Zaganidis

Université Catholique de Louvain, Louvain-la-Neuve, Belgium

O. Bondu, G. Bruno, C. Caputo, P. David, C. Delaere, M. Delcourt, A. Giammanco, V. Lemaitre, A. Magitteri, J. Prisciandaro, A. Saggio, M. Vidal Marono, P. Vischia, J. Zobec

Centro Brasileiro de Pesquisas Fisicas, Rio de Janeiro, Brazil

F.L. Alves, G.A. Alves, G. Correia Silva, C. Hensel, A. Moraes, P. Rebello Teles

Universidade do Estado do Rio de Janeiro, Rio de Janeiro, Brazil

E. Belchior Batista Das Chagas, W. Carvalho, J. Chinellato ${ }^{3}$, E. Coelho, E.M. Da Costa, G.G. Da Silveira ${ }^{4}$, D. De Jesus Damiao, C. De Oliveira Martins, S. Fonseca De Souza, L.M. Huertas Guativa, H. Malbouisson, J. Martins ${ }^{5}$, D. Matos Figueiredo, M. Medina Jaime ${ }^{6}$, M. Melo De Almeida, C. Mora Herrera, L. Mundim, H. Nogima, W.L. Prado Da Silva, L.J. Sanchez Rosas, A. Santoro, A. Sznajder, M. Thiel, E.J. Tonelli Manganote ${ }^{3}$, F. Torres Da Silva De Araujo, A. Vilela Pereira 
Universidade Estadual Paulista ${ }^{a}$, Universidade Federal do $\mathrm{ABC}^{b}$, São Paulo, Brazil

C.A. Bernardes ${ }^{a}$, L. Calligaris ${ }^{a}$, T.R. Fernandez Perez Tomei ${ }^{a}$, E.M. Gregores ${ }^{b}$, D.S. Lemos, P.G. Mercadante ${ }^{b}$, S.F. Novaes ${ }^{a}$, SandraS. Padula ${ }^{a}$

Institute for Nuclear Research and Nuclear Energy, Bulgarian Academy of Sciences, Sofia, Bulgaria

A. Aleksandrov, G. Antchev, R. Hadjiiska, P. Iaydjiev, M. Misheva, M. Rodozov, M. Shopova, G. Sultanov

University of Sofia, Sofia, Bulgaria

M. Bonchev, A. Dimitrov, T. Ivanov, L. Litov, B. Pavlov, P. Petkov

Beihang University, Beijing, China

W. Fang ${ }^{7}, \mathrm{X} . \mathrm{Gao}^{7}, \mathrm{~L}$. Yuan

Institute of High Energy Physics, Beijing, China

G.M. Chen, H.S. Chen, M. Chen, C.H. Jiang, D. Leggat, H. Liao, Z. Liu, A. Spiezia, J. Tao,

E. Yazgan, H. Zhang, S. Zhang ${ }^{8}$, J. Zhao

State Key Laboratory of Nuclear Physics and Technology, Peking University, Beijing, China

A. Agapitos, Y. Ban, G. Chen, A. Levin, J. Li, L. Li, Q. Li, Y. Mao, S.J. Qian, D. Wang, Q. Wang

Tsinghua University, Beijing, China

M. Ahmad, Z. Hu, Y. Wang

Zhejiang University, Hangzhou, China

M. Xiao

Universidad de Los Andes, Bogota, Colombia

C. Avila, A. Cabrera, C. Florez, C.F. González Hernández, M.A. Segura Delgado

Universidad de Antioquia, Medellin, Colombia

J. Mejia Guisao, J.D. Ruiz Alvarez, C.A. Salazar González, N. Vanegas Arbelaez

University of Split, Faculty of Electrical Engineering, Mechanical Engineering and Naval Architecture, Split, Croatia

D. Giljanović, N. Godinovic, D. Lelas, I. Puljak, T. Sculac

University of Split, Faculty of Science, Split, Croatia

Z. Antunovic, M. Kovac

Institute Rudjer Boskovic, Zagreb, Croatia

V. Brigljevic, S. Ceci, D. Ferencek, K. Kadija, B. Mesic, M. Roguljic, A. Starodumov ${ }^{9}$, T. Susa 
University of Cyprus, Nicosia, Cyprus

M.W. Ather, A. Attikis, E. Erodotou, A. Ioannou, M. Kolosova, S. Konstantinou, G. Mavromanolakis, J. Mousa, C. Nicolaou, F. Ptochos, P.A. Razis, H. Rykaczewski, D. Tsiakkouri

Charles University, Prague, Czech Republic

M. Finger ${ }^{10}$, M. Finger Jr. ${ }^{10}$, A. Kveton, J. Tomsa

Escuela Politecnica Nacional, Quito, Ecuador

E. Ayala

Universidad San Francisco de Quito, Quito, Ecuador

E. Carrera Jarrin

Academy of Scientific Research and Technology of the Arab Republic of Egypt, Egyptian Network of High Energy Physics, Cairo, Egypt

S. Abu Zeid ${ }^{11}$, S. Khalil ${ }^{12}$

National Institute of Chemical Physics and Biophysics, Tallinn, Estonia

S. Bhowmik, A. Carvalho Antunes De Oliveira, R.K. Dewanjee, K. Ehataht, M. Kadastik, M. Raidal, C. Veelken

Department of Physics, University of Helsinki, Helsinki, Finland

P. Eerola, L. Forthomme, H. Kirschenmann, K. Osterberg, M. Voutilainen

Helsinki Institute of Physics, Helsinki, Finland

F. Garcia, J. Havukainen, J.K. Heikkilä, T. Järvinen, V. Karimäki, M.S. Kim, R. Kinnunen,

T. Lampén, K. Lassila-Perini, S. Laurila, S. Lehti, T. Lindén, P. Luukka, T. Mäenpää,

H. Siikonen, E. Tuominen, J. Tuominiemi

Lappeenranta University of Technology, Lappeenranta, Finland

T. Tuuva

IRFU, CEA, Université Paris-Saclay, Gif-sur-Yvette, France

M. Besancon, F. Couderc, M. Dejardin, D. Denegri, B. Fabbro, J.L. Faure, F. Ferri, S. Ganjour, A. Givernaud, P. Gras, G. Hamel de Monchenault, P. Jarry, C. Leloup, E. Locci, J. Malcles, J. Rander, A. Rosowsky, M.Ö. Sahin, A. Savoy-Navarro ${ }^{13}$, M. Titov

Laboratoire Leprince-Ringuet, Ecole polytechnique, CNRS/IN2P3, Université Paris-Saclay, Palaiseau, France

S. Ahuja, C. Amendola, F. Beaudette, P. Busson, C. Charlot, B. Diab, G. Falmagne,

R. Granier de Cassagnac, I. Kucher, A. Lobanov, C. Martin Perez, M. Nguyen, C. Ochando,

P. Paganini, J. Rembser, R. Salerno, J.B. Sauvan, Y. Sirois, A. Zabi, A. Zghiche

Université de Strasbourg, CNRS, IPHC UMR 7178, Strasbourg, France

J.-L. Agram ${ }^{14}$, J. Andrea, D. Bloch, G. Bourgatte, J.-M. Brom, E.C. Chabert, C. Collard,

E. Conte ${ }^{14}$, J.-C. Fontaine ${ }^{14}$, D. Gelé, U. Goerlach, M. Jansová, A.-C. Le Bihan, N. Tonon, P. Van Hove 
Centre de Calcul de l'Institut National de Physique Nucleaire et de Physique des Particules, CNRS/IN2P3, Villeurbanne, France

S. Gadrat

Université de Lyon, Université Claude Bernard Lyon 1, CNRS-IN2P3, Institut de Physique Nucléaire de Lyon, Villeurbanne, France

S. Beauceron, C. Bernet, G. Boudoul, C. Camen, A. Carle, N. Chanon, R. Chierici, D. Contardo, P. Depasse, H. El Mamouni, J. Fay, S. Gascon, M. Gouzevitch, B. Ille, Sa. Jain, F. Lagarde, I.B. Laktineh, H. Lattaud, A. Lesauvage, M. Lethuillier, L. Mirabito, S. Perries, V. Sordini, L. Torterotot, G. Touquet, M. Vander Donckt, S. Viret

Georgian Technical University, Tbilisi, Georgia

G. Adamov

Tbilisi State University, Tbilisi, Georgia

Z. Tsamalaidze ${ }^{10}$

RWTH Aachen University, I. Physikalisches Institut, Aachen, Germany

C. Autermann, L. Feld, M.K. Kiesel, K. Klein, M. Lipinski, D. Meuser, A. Pauls, M. Preuten, M.P. Rauch, J. Schulz, M. Teroerde, B. Wittmer

RWTH Aachen University, III. Physikalisches Institut A, Aachen, Germany

A. Albert, M. Erdmann, B. Fischer, S. Ghosh, T. Hebbeker, K. Hoepfner, H. Keller, L. Mastrolorenzo, M. Merschmeyer, A. Meyer, P. Millet, G. Mocellin, S. Mondal, S. Mukherjee, D. Noll, A. Novak, T. Pook, A. Pozdnyakov, T. Quast, M. Radziej, Y. Rath, H. Reithler, J. Roemer, A. Schmidt, S.C. Schuler, A. Sharma, S. Wiedenbeck, S. Zaleski

RWTH Aachen University, III. Physikalisches Institut B, Aachen, Germany

G. Flügge, W. Haj Ahmad ${ }^{15}$, O. Hlushchenko, T. Kress, T. Müller, A. Nehrkorn, A. Nowack, C. Pistone, O. Pooth, D. Roy, H. Sert, A. Stahl ${ }^{16}$

Deutsches Elektronen-Synchrotron, Hamburg, Germany

M. Aldaya Martin, P. Asmuss, I. Babounikau, H. Bakhshiansohi, K. Beernaert, O. Behnke, A. Bermúdez Martínez, D. Bertsche, A.A. Bin Anuar, K. Borras ${ }^{17}$, V. Botta, A. Campbell, A. Cardini, P. Connor, S. Consuegra Rodríguez, C. Contreras-Campana, V. Danilov, A. De Wit, M.M. Defranchis, C. Diez Pardos, D. Domínguez Damiani, G. Eckerlin, D. Eckstein, T. Eichhorn, A. Elwood, E. Eren, E. Gallo ${ }^{18}$, A. Geiser, A. Grohsjean, M. Guthoff, M. Haranko, A. Harb, A. Jafari, N.Z. Jomhari, H. Jung, A. Kasem ${ }^{17}$, M. Kasemann, H. Kaveh, J. Keaveney, C. Kleinwort, J. Knolle, D. Krücker, W. Lange, T. Lenz, J. Lidrych, K. Lipka, W. Lohmann ${ }^{19}$, R. Mankel, I.-A. Melzer-Pellmann, A.B. Meyer, M. Meyer, M. Missiroli, G. Mittag, J. Mnich, A. Mussgiller, V. Myronenko, D. Pérez Adán, S.K. Pflitsch, D. Pitzl, A. Raspereza, A. Saibel, M. Savitskyi, V. Scheurer, P. Schütze, C. Schwanenberger, R. Shevchenko, A. Singh, H. Tholen, O. Turkot, A. Vagnerini, M. Van De Klundert, R. Walsh, Y. Wen, K. Wichmann, C. Wissing, O. Zenaiev, R. Zlebcik University of Hamburg, Hamburg, Germany

R. Aggleton, S. Bein, L. Benato, A. Benecke, V. Blobel, T. Dreyer, A. Ebrahimi, F. Feindt, A. Fröhlich, C. Garbers, E. Garutti, D. Gonzalez, P. Gunnellini, J. Haller, A. Hinzmann, 
A. Karavdina, G. Kasieczka, R. Klanner, R. Kogler, N. Kovalchuk, S. Kurz, V. Kutzner, J. Lange, T. Lange, A. Malara, J. Multhaup, C.E.N. Niemeyer, A. Perieanu, A. Reimers, O. Rieger, C. Scharf, P. Schleper, S. Schumann, J. Schwandt, J. Sonneveld, H. Stadie, G. Steinbrück, F.M. Stober, B. Vormwald, I. Zoi

Karlsruher Institut fuer Technologie, Karlsruhe, Germany

M. Akbiyik, C. Barth, M. Baselga, S. Baur, T. Berger, E. Butz, R. Caspart, T. Chwalek, W. De Boer, A. Dierlamm, K. El Morabit, N. Faltermann, M. Giffels, P. Goldenzweig, A. Gottmann, M.A. Harrendorf, F. Hartmann ${ }^{16}$, U. Husemann, S. Kudella, S. Mitra, M.U. Mozer, D. Müller, Th. Müller, M. Musich, A. Nürnberg, G. Quast, K. Rabbertz, M. Schröder, I. Shvetsov, H.J. Simonis, R. Ulrich, M. Wassmer, M. Weber, C. Wöhrmann, R. Wolf

Institute of Nuclear and Particle Physics (INPP), NCSR Demokritos, Aghia Paraskevi, Greece

G. Anagnostou, P. Asenov, G. Daskalakis, T. Geralis, A. Kyriakis, D. Loukas, G. Paspalaki

National and Kapodistrian University of Athens, Athens, Greece

M. Diamantopoulou, G. Karathanasis, P. Kontaxakis, A. Manousakis-katsikakis, A. Panagiotou, I. Papavergou, N. Saoulidou, A. Stakia, K. Theofilatos, K. Vellidis, E. Vourliotis

National Technical University of Athens, Athens, Greece

G. Bakas, K. Kousouris, I. Papakrivopoulos, G. Tsipolitis

University of Ioánnina, Ioánnina, Greece

I. Evangelou, C. Foudas, P. Gianneios, P. Katsoulis, P. Kokkas, S. Mallios, K. Manitara, N. Manthos, I. Papadopoulos, J. Strologas, F.A. Triantis, D. Tsitsonis

MTA-ELTE Lendület CMS Particle and Nuclear Physics Group, Eötvös Loránd University, Budapest, Hungary

M. Bartók ${ }^{20}$, R. Chudasama, M. Csanad, P. Major, K. Mandal, A. Mehta, M.I. Nagy, G. Pasztor, O. Surányi, G.I. Veres

Wigner Research Centre for Physics, Budapest, Hungary

G. Bencze, C. Hajdu, D. Horvath ${ }^{21}$, F. Sikler, T.Á. Vámi, V. Veszpremi, G. Vesztergombi ${ }^{\dagger}$

Institute of Nuclear Research ATOMKI, Debrecen, Hungary

N. Beni, S. Czellar, J. Karancsi ${ }^{20}$, A. Makovec, J. Molnar, Z. Szillasi

Institute of Physics, University of Debrecen, Debrecen, Hungary

P. Raics, D. Teyssier, Z.L. Trocsanyi, B. Ujvari

Eszterhazy Karoly University, Karoly Robert Campus, Gyongyos, Hungary

T. Csorgo, W.J. Metzger, F. Nemes, T. Novak

Indian Institute of Science (IISc), Bangalore, India

S. Choudhury, J.R. Komaragiri, P.C. Tiwari 
National Institute of Science Education and Research, HBNI, Bhubaneswar, India

S. Bahinipati ${ }^{23}$, C. Kar, G. Kole, P. Mal, V.K. Muraleedharan Nair Bindhu, A. Nayak ${ }^{24}$, D.K. Sahoo ${ }^{23}$, S.K. Swain

Panjab University, Chandigarh, India

S. Bansal, S.B. Beri, V. Bhatnagar, S. Chauhan, R. Chawla, N. Dhingra, R. Gupta,

A. Kaur, M. Kaur, S. Kaur, P. Kumari, M. Lohan, M. Meena, K. Sandeep, S. Sharma, J.B. Singh, A.K. Virdi, G. Walia

University of Delhi, Delhi, India

A. Bhardwaj, B.C. Choudhary, R.B. Garg, M. Gola, S. Keshri, Ashok Kumar, M. Naimuddin, P. Priyanka, K. Ranjan, Aashaq Shah, R. Sharma

Saha Institute of Nuclear Physics, HBNI, Kolkata, India

R. Bhardwaj ${ }^{25}$, M. Bharti ${ }^{25}$, R. Bhattacharya, S. Bhattacharya, U. Bhawandeep ${ }^{25}$, D. Bhowmik, S. Dutta, S. Ghosh, M. Maity ${ }^{26}$, K. Mondal, S. Nandan, A. Purohit, P.K. Rout, G. Saha, S. Sarkar, T. Sarkar ${ }^{26}$, M. Sharan, B. Singh ${ }^{25}$, S. Thakur ${ }^{25}$

Indian Institute of Technology Madras, Madras, India

P.K. Behera, P. Kalbhor, A. Muhammad, P.R. Pujahari, A. Sharma, A.K. Sikdar

Bhabha Atomic Research Centre, Mumbai, India

D. Dutta, V. Jha, V. Kumar, D.K. Mishra, P.K. Netrakanti, L.M. Pant, P. Shukla

Tata Institute of Fundamental Research-A, Mumbai, India

T. Aziz, M.A. Bhat, S. Dugad, G.B. Mohanty, N. Sur, RavindraKumar Verma

Tata Institute of Fundamental Research-B, Mumbai, India

S. Banerjee, S. Bhattacharya, S. Chatterjee, P. Das, M. Guchait, S. Karmakar, S. Kumar,

G. Majumder, K. Mazumdar, N. Sahoo, S. Sawant

Indian Institute of Science Education and Research (IISER), Pune, India

S. Chauhan, S. Dube, V. Hegde, B. Kansal, A. Kapoor, K. Kothekar, S. Pandey, A. Rane, A. Rastogi, S. Sharma

Institute for Research in Fundamental Sciences (IPM), Tehran, Iran

S. Chenarani ${ }^{27}$, E. Eskandari Tadavani, S.M. Etesami ${ }^{27}$, M. Khakzad, M. Mohammadi Najafabadi, M. Naseri, F. Rezaei Hosseinabadi

University College Dublin, Dublin, Ireland

M. Felcini, M. Grunewald

INFN Sezione di Bari ${ }^{a}$, Università di Bari ${ }^{b}$, Politecnico di Bari ${ }^{c}$, Bari, Italy M. Abbrescia ${ }^{a, b}$, R. Aly ${ }^{a, b, 28}$, C. Calabria ${ }^{a, b}$, A. Colaleo ${ }^{a}$, D. Creanza ${ }^{a, c}$, L. Cristella $^{a, b}$, N. De Filippis ${ }^{a, c}$, M. De Palma ${ }^{a, b}$, A. Di Florio ${ }^{a, b}$, W. Elmetenawee ${ }^{a, b}$, L. Fiore $^{a}$,

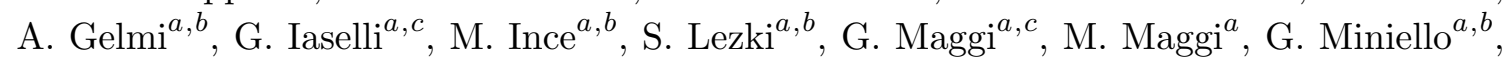
S. $\mathrm{My}^{a, b}$, S. Nuzzo ${ }^{a, b}$, A. Pompili ${ }^{a, b}$, G. Pugliese ${ }^{a, c}$, R. Radogna ${ }^{a}$, A. Ranieri ${ }^{a}$, G. Selvaggi ${ }^{a, b}$, L. Silvestris ${ }^{a}$, F.M. Simone ${ }^{a}$, R. Venditti ${ }^{a}$, P. Verwilligen ${ }^{a}$ 
INFN Sezione di Bologna ${ }^{a}$, Università di Bologna ${ }^{b}$, Bologna, Italy

G. Abbiendi ${ }^{a}$, C. Battilana ${ }^{a, b}$, D. Bonacorsi ${ }^{a, b}$, L. Borgonovi ${ }^{a, b}$, S. Braibant-Giacomelli ${ }^{a, b}$,

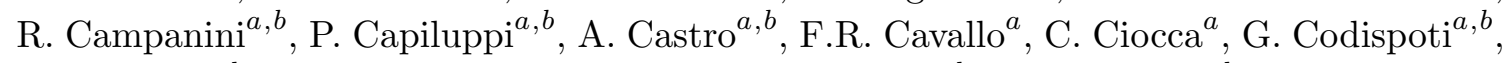
M. Cuffiani ${ }^{a, b}$, G.M. Dallavalle ${ }^{a}$, F. Fabbri ${ }^{a}$, A. Fanfani ${ }^{a, b}$, E. Fontanesi $^{a, b}$, P. Giacomelli $^{a}$, C. Grandi ${ }^{a}$, L. Guiducci ${ }^{a, b}$, F. Iemmi ${ }^{a, b}$, S. Lo Meo ${ }^{a, 29}$, S. Marcellini ${ }^{a}$, G. Masetti ${ }^{a}$,

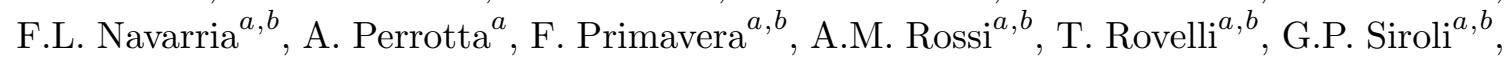
N. $\operatorname{Tosi}^{a}$

INFN Sezione di Catania $^{a}$, Università di Catania ${ }^{b}$, Catania, Italy

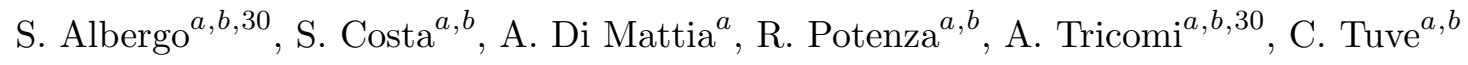

INFN Sezione di Firenze ${ }^{a}$, Università di Firenze ${ }^{b}$, Firenze, Italy

G. Barbagli ${ }^{a}$, A. Cassese, R. Ceccarelli, V. Ciulli ${ }^{a, b}$, C. Civinini $^{a}$, R. D'Alessandro ${ }^{a, b}{ }$ E. Focardi ${ }^{a, b}$, G. Latino ${ }^{a, b}$, P. Lenzi ${ }^{a, b}$, M. Meschini ${ }^{a}$, S. Paoletti ${ }^{a}$, G. Sguazzoni ${ }^{a}$, L. Viliani ${ }^{a}$

INFN Laboratori Nazionali di Frascati, Frascati, Italy

L. Benussi, S. Bianco, D. Piccolo

INFN Sezione di Genova ${ }^{a}$, Università di Genova ${ }^{b}$, Genova, Italy

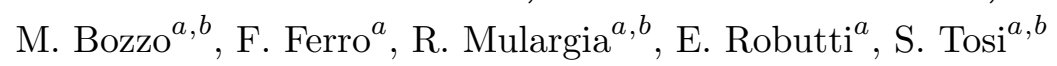

INFN Sezione di Milano-Bicocca ${ }^{a}$, Università di Milano-Bicocca ${ }^{b}$, Milano, Italy

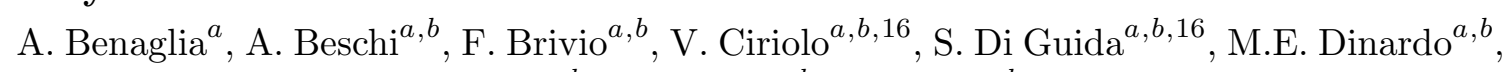
P. Dini ${ }^{a}$, S. Gennai ${ }^{a}$, A. Ghezzi ${ }^{a, b}$, P. Govoni ${ }^{a}, b$, L. Guzzi $^{a, b}$, M. Malberti $^{a}$, S. Malvezzi $^{a}$, D. Menasce ${ }^{a}$, F. Monti ${ }^{a, b}$, L. Moroni ${ }^{a}$, M. Paganoni ${ }^{a, b}$, D. Pedrini ${ }^{a}$, S. Ragazzi ${ }^{a, b}$, T. Tabarelli de Fatis ${ }^{a, b}$, D. Zuolo ${ }^{a, b}$

INFN Sezione di Napoli ${ }^{a}$, Università di Napoli 'Federico II' ${ }^{b}$, Napoli, Italy, Università della Basilicata $^{c}$, Potenza, Italy, Università G. Marconi ${ }^{d}$, Roma, Italy

S. Buontempo ${ }^{a}$, N. Cavallo ${ }^{a, c}$, A. De Iorio ${ }^{a, b}$, A. Di Crescenzo ${ }^{a, b}$, F. Fabozzi $^{a, c}$, F. Fienga $^{a}$, G. Galati ${ }^{a}$, A.O.M. Iorio ${ }^{a, b}$, L. Lista ${ }^{a, b}$, S. Meola ${ }^{a, d, 16}$, P. Paolucci ${ }^{a, 16}$, B. Rossi ${ }^{a}$, C. Sciacca ${ }^{a, b}$, E. Voevodina ${ }^{a, b}$

INFN Sezione di Padova ${ }^{a}$, Università di Padova ${ }^{b}$, Padova, Italy, Università di Trento ${ }^{c}$, Trento, Italy

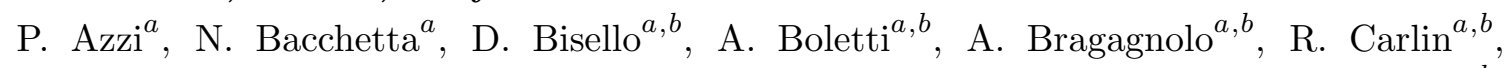

P. Checchia ${ }^{a}$, P. De Castro Manzano ${ }^{a}$, T. Dorigo ${ }^{a}$, U. Dosselli ${ }^{a}$, F. Gasparini ${ }^{a, b}$,

U. Gasparini $^{a, b}$, A. Gozzelino ${ }^{a}$, S.Y. Hoh $^{a, b}$, P. Lujan ${ }^{a}$, M. Margoni $^{a, b}$, A.T. Meneguzzo ${ }^{a, b}$,

J. Pazzini ${ }^{a, b}$, M. Presilla ${ }^{b}$, P. Ronchese ${ }^{a, b}$, R. Rossin ${ }^{a, b}$, F. Simonetto ${ }^{a, b}$, A. Tiko ${ }^{a}$, M. Tosi ${ }^{a, b}$, M. Zanetti ${ }^{a, b}$, P. Zotto ${ }^{a, b}$, G. Zumerle ${ }^{a, b}$

INFN Sezione di Pavia ${ }^{a}$, Università di Pavia ${ }^{b}$, Pavia, Italy

A. Braghieri ${ }^{a}$, D. Fiorina ${ }^{a, b}$, P. Montagna ${ }^{a, b}$, S.P. Ratti ${ }^{a, b}$, V. $\operatorname{Re}^{a}$, M. Ressegotti ${ }^{a, b}$,

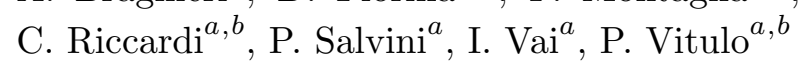


INFN Sezione di Perugia ${ }^{a}$, Università di Perugia ${ }^{b}$, Perugia, Italy

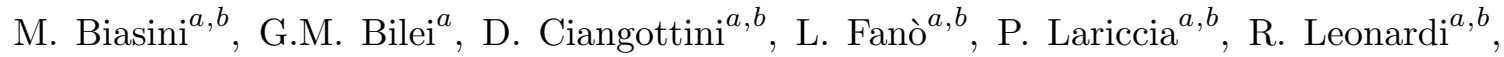

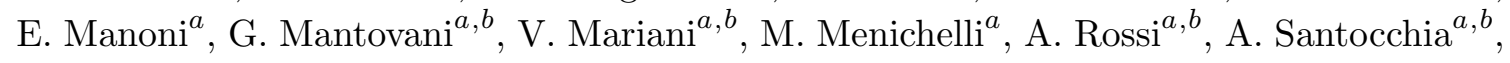

D. Spiga ${ }^{a}$

INFN Sezione di Pisa ${ }^{a}$, Università di Pisa ${ }^{b}$, Scuola Normale Superiore di Pisa c, Pisa, Italy

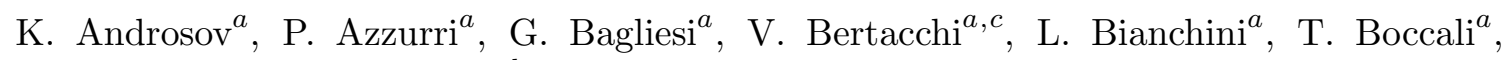
R. Castaldi $^{a}$, M.A. Ciocci ${ }^{a, b}$, R. Dell'Orso ${ }^{a}$, G. Fedi ${ }^{a}$, L. Giannini ${ }^{a, c}$, A. Giassi $^{a}$,

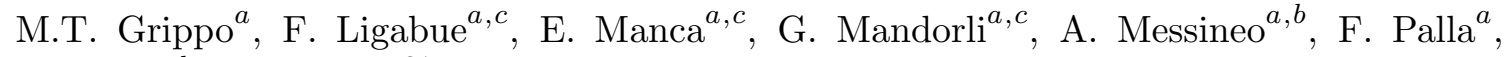
A. Rizzi ${ }^{a}, b$ G. Rolandi ${ }^{31}$, S. Roy Chowdhury, A. Scribano ${ }^{a}$, P. Spagnolo $^{a}$, R. Tenchini ${ }^{a}$, G. Tonelli ${ }^{a, b}$, N. Turini, A. Venturi ${ }^{a}$, P.G. Verdini ${ }^{a}$

INFN Sezione di Roma ${ }^{a}$, Sapienza Università di Roma ${ }^{b}$, Rome, Italy

F. Cavallari ${ }^{a}$, M. Cipriani ${ }^{a, b}$, D. Del Re ${ }^{a, b}$, E. Di Marco ${ }^{a, b}$, M. Diemoz $^{a}$, E. Longo ${ }^{a, b}$, P. Meridiani ${ }^{a}$, G. Organtini ${ }^{a, b}$, F. Pandolfi ${ }^{a}$, R. Paramatti ${ }^{a, b}$, C. Quaranta ${ }^{a, b}$, S. Rahatlou ${ }^{a, b}$, C. Rovelli ${ }^{a}$, F. Santanastasio ${ }^{a, b}$, L. Soffi $^{a, b}$

INFN Sezione di Torino ${ }^{a}$, Università di Torino ${ }^{b}$, Torino, Italy, Università del Piemonte Orientale ${ }^{c}$, Novara, Italy

N. Amapane ${ }^{a, b}$, R. Arcidiacono ${ }^{a, c}, \mathrm{~S}$. Argiro $^{a, b}$, M. Arneodo $^{a, c}, \mathrm{~N}_{\text {Bartosik }}{ }^{a}$, R. Bellan $^{a, b}$, A. Bellora, C. Biino ${ }^{a}$, A. Cappati ${ }^{a, b}$, N. Cartiglia ${ }^{a}$, S. Cometti ${ }^{a}$, M. Costa $^{a, b}$, R. Covarelli ${ }^{a, b}$, N. Demaria ${ }^{a}$, B. Kiani ${ }^{a, b}$, C. Mariotti ${ }^{a}$, S. Maselli ${ }^{a}$, E. Migliore ${ }^{a, b}$, V. Monaco ${ }^{a, b}$, E. Monteil $^{a, b}$, M. Monteno $^{a}$, M.M. Obertino ${ }^{a, b}$, G. Ortona $^{a, b}$, L. Pacher ${ }^{a, b}$, N. Pastrone $^{a}$, M. Pelliccioni ${ }^{a}$, G.L. Pinna Angioni ${ }^{a, b}$, A. Romero ${ }^{a, b}$, M. Ruspa ${ }^{a, c}$, R. Salvatico ${ }^{a, b}$, V. Sola ${ }^{a}$, A. Solano ${ }^{a, b}$, D. Soldi ${ }^{a, b}$, A. Staiano ${ }^{a}$

INFN Sezione di Trieste ${ }^{a}$, Università di Trieste ${ }^{b}$, Trieste, Italy

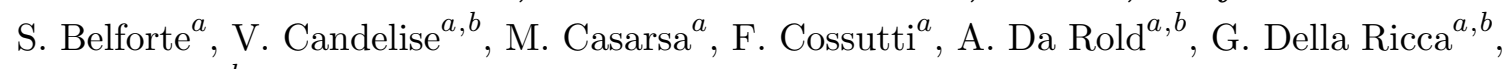
F. Vazzoler ${ }^{a, b}$, A. Zanetti ${ }^{a}$

Kyungpook National University, Daegu, Korea

B. Kim, D.H. Kim, G.N. Kim, J. Lee, S.W. Lee, C.S. Moon, Y.D. Oh, S.I. Pak, S. Sekmen, D.C. Son, Y.C. Yang

Chonnam National University, Institute for Universe and Elementary Particles, Kwangju, Korea

H. Kim, D.H. Moon, G. Oh

Hanyang University, Seoul, Korea

B. Francois, T.J. Kim, J. Park

Korea University, Seoul, Korea

S. Cho, S. Choi, Y. Go, D. Gyun, S. Ha, B. Hong, K. Lee, K.S. Lee, J. Lim, J. Park, S.K. Park, Y. Roh, J. Yoo

Kyung Hee University, Department of Physics

J. Goh 
Sejong University, Seoul, Korea

H.S. Kim

Seoul National University, Seoul, Korea

J. Almond, J.H. Bhyun, J. Choi, S. Jeon, J. Kim, J.S. Kim, H. Lee, K. Lee, S. Lee, K. Nam, M. Oh, S.B. Oh, B.C. Radburn-Smith, U.K. Yang, H.D. Yoo, I. Yoon, G.B. Yu

University of Seoul, Seoul, Korea

D. Jeon, H. Kim, J.H. Kim, J.S.H. Lee, I.C. Park, I.J Watson

Sungkyunkwan University, Suwon, Korea

Y. Choi, C. Hwang, Y. Jeong, J. Lee, Y. Lee, I. Yu

Riga Technical University, Riga, Latvia

V. Veckalns ${ }^{32}$

Vilnius University, Vilnius, Lithuania

V. Dudenas, A. Juodagalvis, G. Tamulaitis, J. Vaitkus

National Centre for Particle Physics, Universiti Malaya, Kuala Lumpur, Malaysia

Z.A. Ibrahim, F. Mohamad Idris ${ }^{33}$, W.A.T. Wan Abdullah, M.N. Yusli, Z. Zolkapli

Universidad de Sonora (UNISON), Hermosillo, Mexico

J.F. Benitez, A. Castaneda Hernandez, J.A. Murillo Quijada, L. Valencia Palomo

Centro de Investigacion y de Estudios Avanzados del IPN, Mexico City, Mexico

H. Castilla-Valdez, E. De La Cruz-Burelo, I. Heredia-De La Cruz ${ }^{34}$, R. Lopez-Fernandez,

A. Sanchez-Hernandez

Universidad Iberoamericana, Mexico City, Mexico

S. Carrillo Moreno, C. Oropeza Barrera, M. Ramirez-Garcia, F. Vazquez Valencia

Benemerita Universidad Autonoma de Puebla, Puebla, Mexico

J. Eysermans, I. Pedraza, H.A. Salazar Ibarguen, C. Uribe Estrada

Universidad Autónoma de San Luis Potosí, San Luis Potosí, Mexico

A. Morelos Pineda

University of Montenegro, Podgorica, Montenegro

J. Mijuskovic, N. Raicevic

University of Auckland, Auckland, New Zealand

D. Krofcheck

University of Canterbury, Christchurch, New Zealand

S. Bheesette, P.H. Butler

National Centre for Physics, Quaid-I-Azam University, Islamabad, Pakistan

A. Ahmad, M. Ahmad, Q. Hassan, H.R. Hoorani, W.A. Khan, M.A. Shah, M. Shoaib, M. Waqas 
AGH University of Science and Technology Faculty of Computer Science, Electronics and Telecommunications, Krakow, Poland

V. Avati, L. Grzanka, M. Malawski

National Centre for Nuclear Research, Swierk, Poland

H. Bialkowska, M. Bluj, B. Boimska, M. Górski, M. Kazana, M. Szleper, P. Zalewski

Institute of Experimental Physics, Faculty of Physics, University of Warsaw, Warsaw, Poland

K. Bunkowski, A. Byszuk ${ }^{35}$, K. Doroba, A. Kalinowski, M. Konecki, J. Krolikowski, M. Misiura, M. Olszewski, M. Walczak

Laboratório de Instrumentação e Física Experimental de Partículas, Lisboa, Portugal

M. Araujo, P. Bargassa, D. Bastos, A. Di Francesco, P. Faccioli, B. Galinhas, M. Gallinaro, J. Hollar, N. Leonardo, T.S. Niknejad, J. Seixas, K. Shchelina, G. Strong, O. Toldaiev, J. Varela

Joint Institute for Nuclear Research, Dubna, Russia

S. Afanasiev, P. Bunin, M. Gavrilenko, I. Golutvin, I. Gorbunov, A. Kamenev, V. Karjavine, A. Lanev, A. Malakhov, V. Matveev ${ }^{36,37}$, P. Moisenz, V. Palichik, V. Perelygin, M. Savina, S. Shmatov, S. Shulha, N. Skatchkov, V. Smirnov, N. Voytishin, A. Zarubin

Petersburg Nuclear Physics Institute, Gatchina (St. Petersburg), Russia

L. Chtchipounov, V. Golovtcov, Y. Ivanov, V. Kim ${ }^{38}$, E. Kuznetsova ${ }^{39}$, P. Levchenko, V. Murzin, V. Oreshkin, I. Smirnov, D. Sosnov, V. Sulimov, L. Uvarov, A. Vorobyev

Institute for Nuclear Research, Moscow, Russia

Yu. Andreev, A. Dermenev, S. Gninenko, N. Golubev, A. Karneyeu, M. Kirsanov, N. Krasnikov, A. Pashenkov, D. Tlisov, A. Toropin

Institute for Theoretical and Experimental Physics named by A.I. Alikhanov of NRC 'Kurchatov Institute', Moscow, Russia

V. Epshteyn, V. Gavrilov, N. Lychkovskaya, A. Nikitenko ${ }^{40}$, V. Popov, I. Pozdnyakov, G. Safronov, A. Spiridonov, A. Stepennov, M. Toms, E. Vlasov, A. Zhokin

Moscow Institute of Physics and Technology, Moscow, Russia

T. Aushev

National Research Nuclear University 'Moscow Engineering Physics Institute' (MEPhI), Moscow, Russia

M. Chadeeva ${ }^{41}$, P. Parygin, D. Philippov, V. Rusinov, E. Zhemchugov

P.N. Lebedev Physical Institute, Moscow, Russia

V. Andreev, M. Azarkin, I. Dremin, M. Kirakosyan, A. Terkulov 
Skobeltsyn Institute of Nuclear Physics, Lomonosov Moscow State University, Moscow, Russia

A. Baskakov, A. Belyaev, E. Boos, V. Bunichev, M. Dubinin ${ }^{42}$, L. Dudko, A. Ershov, V. Klyukhin, O. Kodolova, I. Lokhtin, S. Obraztsov, S. Petrushanko, V. Savrin

Novosibirsk State University (NSU), Novosibirsk, Russia

A. Barnyakov ${ }^{43}$, V. Blinov ${ }^{43}$, T. Dimova ${ }^{43}$, L. Kardapoltsev ${ }^{43}$, Y. Skovpen ${ }^{43}$

Institute for High Energy Physics of National Research Centre 'Kurchatov Institute', Protvino, Russia

I. Azhgirey, I. Bayshev, S. Bitioukov, V. Kachanov, D. Konstantinov, P. Mandrik,

V. Petrov, R. Ryutin, S. Slabospitskii, A. Sobol, S. Troshin, N. Tyurin, A. Uzunian,

A. Volkov

National Research Tomsk Polytechnic University, Tomsk, Russia

A. Babaev, A. Iuzhakov, V. Okhotnikov

Tomsk State University, Tomsk, Russia

V. Borchsh, V. Ivanchenko, E. Tcherniaev

University of Belgrade: Faculty of Physics and VINCA Institute of Nuclear Sciences

P. Adzic ${ }^{44}$, P. Cirkovic, D. Devetak, M. Dordevic, P. Milenovic, J. Milosevic, M. Stojanovic

Centro de Investigaciones Energéticas Medioambientales y Tecnológicas (CIEMAT), Madrid, Spain

M. Aguilar-Benitez, J. Alcaraz Maestre, A. Álvarez Fernández, I. Bachiller, M. Barrio Luna, J.A. Brochero Cifuentes, C.A. Carrillo Montoya, M. Cepeda, M. Cerrada, N. Colino, B. De La Cruz, A. Delgado Peris, C. Fernandez Bedoya, J.P. Fernández Ramos, J. Flix, M.C. Fouz, O. Gonzalez Lopez, S. Goy Lopez, J.M. Hernandez, M.I. Josa, D. Moran, Á. Navarro Tobar, A. Pérez-Calero Yzquierdo, J. Puerta Pelayo, I. Redondo, L. Romero, S. Sánchez Navas, M.S. Soares, A. Triossi, C. Willmott

Universidad Autónoma de Madrid, Madrid, Spain

C. Albajar, J.F. de Trocóniz, R. Reyes-Almanza

Universidad de Oviedo, Instituto Universitario de Ciencias y Tecnologías Espaciales de Asturias (ICTEA), Oviedo, Spain

B. Alvarez Gonzalez, J. Cuevas, C. Erice, J. Fernandez Menendez, S. Folgueras, I. Gonzalez Caballero, J.R. González Fernández, E. Palencia Cortezon, V. Rodríguez Bouza, S. Sanchez Cruz

Instituto de Física de Cantabria (IFCA), CSIC-Universidad de Cantabria, Santander, Spain

I.J. Cabrillo, A. Calderon, B. Chazin Quero, J. Duarte Campderros, M. Fernandez, P.J. Fernández Manteca, A. García Alonso, G. Gomez, C. Martinez Rivero, P. Martinez Ruiz del Arbol, F. Matorras, J. Piedra Gomez, C. Prieels, T. Rodrigo, A. Ruiz-Jimeno, L. Russo $^{45}$, L. Scodellaro, N. Trevisani, I. Vila, J.M. Vizan Garcia 


\section{University of Colombo, Colombo, Sri Lanka}

K. Malagalage

\section{University of Ruhuna, Department of Physics, Matara, Sri Lanka}

W.G.D. Dharmaratna, N. Wickramage

\section{CERN, European Organization for Nuclear Research, Geneva, Switzerland}

D. Abbaneo, B. Akgun, E. Auffray, G. Auzinger, J. Baechler, P. Baillon, A.H. Ball, D. Barney, J. Bendavid, M. Bianco, A. Bocci, P. Bortignon, E. Bossini, C. Botta, E. Brondolin, T. Camporesi, A. Caratelli, G. Cerminara, E. Chapon, G. Cucciati, D. d'Enterria, A. Dabrowski, N. Daci, V. Daponte, A. David, O. Davignon, A. De Roeck, M. Deile, M. Dobson, M. Dünser, N. Dupont, A. Elliott-Peisert, N. Emriskova, F. Fallavollita ${ }^{46}$, D. Fasanella, S. Fiorendi, G. Franzoni, J. Fulcher, W. Funk, S. Giani, D. Gigi, A. Gilbert, K. Gill, F. Glege, M. Gruchala, M. Guilbaud, D. Gulhan, J. Hegeman, C. Heidegger, Y. Iiyama, V. Innocente, P. Janot, O. Karacheban ${ }^{19}$, J. Kaspar, J. Kieseler, M. Krammer ${ }^{1}$, N. Kratochwil, C. Lange, P. Lecoq, C. Lourenço, L. Malgeri, M. Mannelli, A. Massironi, F. Meijers, J.A. Merlin, S. Mersi, E. Meschi, F. Moortgat, M. Mulders, J. Ngadiuba, J. Niedziela, S. Nourbakhsh, S. Orfanelli, L. Orsini, F. Pantaleo ${ }^{16}$, L. Pape, E. Perez, M. Peruzzi, A. Petrilli, G. Petrucciani, A. Pfeiffer, M. Pierini, F.M. Pitters, D. Rabady, A. Racz, M. Rieger, M. Rovere, H. Sakulin, C. Schäfer, C. Schwick, M. Selvaggi, A. Sharma, P. Silva, W. Snoeys, P. Sphicas ${ }^{47}$, J. Steggemann, S. Summers, V.R. Tavolaro, D. Treille, A. Tsirou, G.P. Van Onsem, A. Vartak, M. Verzetti, W.D. Zeuner

\section{Paul Scherrer Institut, Villigen, Switzerland}

L. Caminada ${ }^{48}$, K. Deiters, W. Erdmann, R. Horisberger, Q. Ingram, H.C. Kaestli, D. Kotlinski, U. Langenegger, T. Rohe, S.A. Wiederkehr

ETH Zurich - Institute for Particle Physics and Astrophysics (IPA), Zurich, Switzerland

M. Backhaus, P. Berger, N. Chernyavskaya, G. Dissertori, M. Dittmar, M. Donegà, C. Dorfer, T.A. Gómez Espinosa, C. Grab, D. Hits, T. Klijnsma, W. Lustermann, R.A. Manzoni, M. Marionneau, M.T. Meinhard, F. Micheli, P. Musella, F. Nessi-Tedaldi, F. Pauss, G. Perrin, L. Perrozzi, S. Pigazzini, M.G. Ratti, M. Reichmann, C. Reissel, T. Reitenspiess, D. Ruini, D.A. Sanz Becerra, M. Schönenberger, L. Shchutska, M.L. Vesterbacka Olsson, R. Wallny, D.H. Zhu

\section{Universität Zürich, Zurich, Switzerland}

T.K. Aarrestad, C. Amsler ${ }^{49}$, D. Brzhechko, M.F. Canelli, A. De Cosa, R. Del Burgo, S. Donato, B. Kilminster, S. Leontsinis, V.M. Mikuni, I. Neutelings, G. Rauco, P. Robmann, D. Salerno, K. Schweiger, C. Seitz, Y. Takahashi, S. Wertz, A. Zucchetta

\section{National Central University, Chung-Li, Taiwan}

T.H. Doan, C.M. Kuo, W. Lin, A. Roy, S.S. Yu

\section{National Taiwan University (NTU), Taipei, Taiwan}

P. Chang, Y. Chao, K.F. Chen, P.H. Chen, W.-S. Hou, Y.y. Li, R.-S. Lu, E. Paganis, A. Psallidas, A. Steen 
Chulalongkorn University, Faculty of Science, Department of Physics, Bangkok, Thailand

B. Asavapibhop, C. Asawatangtrakuldee, N. Srimanobhas, N. Suwonjandee

Çukurova University, Physics Department, Science and Art Faculty, Adana, Turkey

A. Bat, F. Boran, A. Celik $^{50}$, S. Cerci $^{51}$, S. Damarseckin ${ }^{52}$, Z.S. Demiroglu, F. Dolek, C. Dozen ${ }^{53}$, I. Dumanoglu, G. Gokbulut, EmineGurpinar Guler ${ }^{54}$, Y. Guler, I. Hos ${ }^{55}$, C. Isik, E.E. Kangal ${ }^{56}$, O. Kara, A. Kayis Topaksu, U. Kiminsu, G. Onengut, K. Ozdemir ${ }^{57}$, S. Ozturk ${ }^{58}$, A.E. Simsek, D. Sunar Cerci ${ }^{51}$, U.G. Tok, S. Turkcapar, I.S. Zorbakir, C. Zorbilmez

Middle East Technical University, Physics Department, Ankara, Turkey B. Isildak ${ }^{59}$, G. Karapinar ${ }^{60}$, M. Yalvac

Bogazici University, Istanbul, Turkey

I.O. Atakisi, E. Gülmez, M. Kaya ${ }^{61}$, O. Kaya ${ }^{62}$, Ö. Özçelik, S. Tekten, E.A. Yetkin ${ }^{63}$

Istanbul Technical University, Istanbul, Turkey

A. Cakir, K. Cankocak, Y. Komurcu, S. Sen ${ }^{64}$

Istanbul University, Istanbul, Turkey

B. Kaynak, S. Ozkorucuklu

Institute for Scintillation Materials of National Academy of Science of Ukraine, Kharkov, Ukraine

B. Grynyov

National Scientific Center, Kharkov Institute of Physics and Technology, Kharkov, Ukraine

L. Levchuk

University of Bristol, Bristol, United Kingdom

E. Bhal, S. Bologna, J.J. Brooke, D. Burns ${ }^{65}$, E. Clement, D. Cussans, H. Flacher, J. Goldstein, G.P. Heath, H.F. Heath, L. Kreczko, S. Paramesvaran, B. Penning, T. Sakuma, S. Seif El Nasr-Storey, V.J. Smith, J. Taylor, A. Titterton

\section{Rutherford Appleton Laboratory, Didcot, United Kingdom}

K.W. Bell, A. Belyaev ${ }^{66}$, C. Brew, R.M. Brown, D. Cieri, D.J.A. Cockerill, J.A. Coughlan, K. Harder, S. Harper, J. Linacre, K. Manolopoulos, D.M. Newbold, E. Olaiya, D. Petyt, T. Reis, T. Schuh, C.H. Shepherd-Themistocleous, A. Thea, I.R. Tomalin, T. Williams, W.J. Womersley

\section{Imperial College, London, United Kingdom}

R. Bainbridge, P. Bloch, J. Borg, S. Breeze, O. Buchmuller, A. Bundock, GurpreetSingh CHAHAL ${ }^{67}$, D. Colling, P. Dauncey, G. Davies, M. Della Negra, R. Di Maria, P. Everaerts, G. Hall, G. Iles, T. James, M. Komm, C. Laner, L. Lyons, A.-M. Magnan, S. Malik, A. Martelli, V. Milosevic, J. Nash ${ }^{68}$, V. Palladino, M. Pesaresi, D.M. Raymond, 
A. Richards, A. Rose, E. Scott, C. Seez, A. Shtipliyski, M. Stoye, T. Strebler, A. Tapper, K. Uchida, T. Virdee ${ }^{16}$, N. Wardle, D. Winterbottom, J. Wright, A.G. Zecchinelli, S.C. Zenz

\section{Brunel University, Uxbridge, United Kingdom}

J.E. Cole, P.R. Hobson, A. Khan, P. Kyberd, C.K. Mackay, A. Morton, I.D. Reid, L. Teodorescu, S. Zahid

\section{Baylor University, Waco, U.S.A.}

K. Call, B. Caraway, J. Dittmann, K. Hatakeyama, C. Madrid, B. McMaster, N. Pastika, C. Smith

\section{Catholic University of America, Washington, DC, U.S.A.}

R. Bartek, A. Dominguez, R. Uniyal, A.M. Vargas Hernandez

\section{The University of Alabama, Tuscaloosa, U.S.A.}

A. Buccilli, S.I. Cooper, C. Henderson, P. Rumerio, C. West

\section{Boston University, Boston, U.S.A.}

D. Arcaro, Z. Demiragli, D. Gastler, C. Richardson, J. Rohlf, D. Sperka, I. Suarez, L. Sulak, D. Zou

\section{Brown University, Providence, U.S.A.}

G. Benelli, B. Burkle, X. Coubez ${ }^{17}$, D. Cutts, Y.t. Duh, M. Hadley, J. Hakala, U. Heintz, J.M. $\operatorname{Hogan}^{69}$, K.H.M. Kwok, E. Laird, G. Landsberg, J. Lee, Z. Mao, M. Narain, S. Sagir ${ }^{70}$, R. Syarif, E. Usai, D. Yu, W. Zhang

\section{University of California, Davis, Davis, U.S.A.}

R. Band, C. Brainerd, R. Breedon, M. Calderon De La Barca Sanchez, M. Chertok, J. Conway, R. Conway, P.T. Cox, R. Erbacher, C. Flores, G. Funk, F. Jensen, W. Ko, O. Kukral, R. Lander, M. Mulhearn, D. Pellett, J. Pilot, M. Shi, D. Taylor, K. Tos, M. Tripathi, Z. Wang, F. Zhang

\section{University of California, Los Angeles, U.S.A.}

M. Bachtis, C. Bravo, R. Cousins, A. Dasgupta, A. Florent, J. Hauser, M. Ignatenko, N. Mccoll, W.A. Nash, S. Regnard, D. Saltzberg, C. Schnaible, B. Stone, V. Valuev

University of California, Riverside, Riverside, U.S.A.

K. Burt, Y. Chen, R. Clare, J.W. Gary, S.M.A. Ghiasi Shirazi, G. Hanson, G. Karapostoli, E. Kennedy, O.R. Long, M. Olmedo Negrete, M.I. Paneva, W. Si, L. Wang, S. Wimpenny, B.R. Yates, Y. Zhang

\section{University of California, San Diego, La Jolla, U.S.A.}

J.G. Branson, P. Chang, S. Cittolin, S. Cooperstein, N. Deelen, M. Derdzinski, R. Gerosa, D. Gilbert, B. Hashemi, D. Klein, V. Krutelyov, J. Letts, M. Masciovecchio, S. May, S. Padhi, M. Pieri, V. Sharma, M. Tadel, F. Würthwein, A. Yagil, G. Zevi Della Porta 
University of California, Santa Barbara - Department of Physics, Santa Barbara, U.S.A.

N. Amin, R. Bhandari, C. Campagnari, M. Citron, V. Dutta, M. Franco Sevilla, L. Gouskos, J. Incandela, B. Marsh, H. Mei, A. Ovcharova, H. Qu, J. Richman, U. Sarica, D. Stuart, S. Wang

\section{California Institute of Technology, Pasadena, U.S.A.}

D. Anderson, A. Bornheim, O. Cerri, I. Dutta, J.M. Lawhorn, N. Lu, J. Mao, H.B. Newman, T.Q. Nguyen, J. Pata, M. Spiropulu, J.R. Vlimant, S. Xie, Z. Zhang, R.Y. Zhu

Carnegie Mellon University, Pittsburgh, U.S.A.

M.B. Andrews, T. Ferguson, T. Mudholkar, M. Paulini, M. Sun, I. Vorobiev, M. Weinberg

University of Colorado Boulder, Boulder, U.S.A.

J.P. Cumalat, W.T. Ford, A. Johnson, E. MacDonald, T. Mulholland, R. Patel, A. Perloff, K. Stenson, K.A. Ulmer, S.R. Wagner

Cornell University, Ithaca, U.S.A.

J. Alexander, J. Chaves, Y. Cheng, J. Chu, A. Datta, A. Frankenthal, K. Mcdermott, J.R. Patterson, D. Quach, A. Rinkevicius ${ }^{71}$, A. Ryd, S.M. Tan, Z. Tao, J. Thom, P. Wittich, M. Zientek

Fermi National Accelerator Laboratory, Batavia, U.S.A.

S. Abdullin, M. Albrow, M. Alyari, G. Apollinari, A. Apresyan, A. Apyan, S. Banerjee, L.A.T. Bauerdick, A. Beretvas, D. Berry, J. Berryhill, P.C. Bhat, K. Burkett, J.N. Butler, A. Canepa, G.B. Cerati, H.W.K. Cheung, F. Chlebana, M. Cremonesi, J. Duarte, V.D. Elvira, J. Freeman, Z. Gecse, E. Gottschalk, L. Gray, D. Green, S. Grünendahl, O. Gutsche, AllisonReinsvold Hall, J. Hanlon, R.M. Harris, S. Hasegawa, R. Heller, J. Hirschauer, B. Jayatilaka, S. Jindariani, M. Johnson, U. Joshi, B. Klima, M.J. Kortelainen, B. Kreis, S. Lammel, J. Lewis, D. Lincoln, R. Lipton, M. Liu, T. Liu, J. Lykken, K. Maeshima, J.M. Marraffino, D. Mason, P. McBride, P. Merkel, S. Mrenna, S. Nahn, V. O'Dell, V. Papadimitriou, K. Pedro, C. Pena, G. Rakness, F. Ravera, L. Ristori, B. Schneider, E. Sexton-Kennedy, N. Smith, A. Soha, W.J. Spalding, L. Spiegel, S. Stoynev, J. Strait, N. Strobbe, L. Taylor, S. Tkaczyk, N.V. Tran, L. Uplegger, E.W. Vaandering, C. Vernieri, R. Vidal, M. Wang, H.A. Weber

University of Florida, Gainesville, U.S.A.

D. Acosta, P. Avery, D. Bourilkov, A. Brinkerhoff, L. Cadamuro, A. Carnes, V. Cherepanov, F. Errico, R.D. Field, S.V. Gleyzer, B.M. Joshi, M. Kim, J. Konigsberg, A. Korytov, K.H. Lo, P. Ma, K. Matchev, N. Menendez, G. Mitselmakher, D. Rosenzweig, K. Shi, J. Wang, S. Wang, X. Zuo

Florida International University, Miami, U.S.A.

Y.R. Joshi

Florida State University, Tallahassee, U.S.A.

T. Adams, A. Askew, S. Hagopian, V. Hagopian, K.F. Johnson, R. Khurana, T. Kolberg, G. Martinez, T. Perry, H. Prosper, C. Schiber, R. Yohay, J. Zhang 
Florida Institute of Technology, Melbourne, U.S.A.

M.M. Baarmand, M. Hohlmann, D. Noonan, M. Rahmani, M. Saunders, F. Yumiceva

University of Illinois at Chicago (UIC), Chicago, U.S.A.

M.R. Adams, L. Apanasevich, R.R. Betts, R. Cavanaugh, X. Chen, S. Dittmer, O. Evdokimov, C.E. Gerber, D.A. Hangal, D.J. Hofman, K. Jung, C. Mills, T. Roy, M.B. Tonjes, N. Varelas, J. Viinikainen, H. Wang, X. Wang, Z. Wu

The University of Iowa, Iowa City, U.S.A.

M. Alhusseini, B. Bilki ${ }^{54}$, W. Clarida, K. Dilsiz ${ }^{72}$, S. Durgut, R.P. Gandrajula, M. Haytmyradov, V. Khristenko, O.K. Köseyan, J.-P. Merlo, A. Mestvirishvili ${ }^{73}$, A. Moeller, J. Nachtman, H. Ogul ${ }^{74}$, Y. Onel, F. Ozok ${ }^{75}$, A. Penzo, C. Snyder, E. Tiras, J. Wetzel

Johns Hopkins University, Baltimore, U.S.A.

B. Blumenfeld, A. Cocoros, N. Eminizer, A.V. Gritsan, W.T. Hung, S. Kyriacou, P. Maksimovic, J. Roskes, M. Swartz

The University of Kansas, Lawrence, U.S.A.

C. Baldenegro Barrera, P. Baringer, A. Bean, S. Boren, J. Bowen, A. Bylinkin,

T. Isidori, S. Khalil, J. King, G. Krintiras, A. Kropivnitskaya, C. Lindsey, D. Majumder,

W. Mcbrayer, N. Minafra, M. Murray, C. Rogan, C. Royon, S. Sanders, E. Schmitz, J.D. Tapia Takaki, Q. Wang, J. Williams, G. Wilson

Kansas State University, Manhattan, U.S.A.

S. Duric, A. Ivanov, K. Kaadze, D. Kim, Y. Maravin, D.R. Mendis, T. Mitchell, A. Modak, A. Mohammadi

Lawrence Livermore National Laboratory, Livermore, U.S.A.

F. Rebassoo, D. Wright

University of Maryland, College Park, U.S.A.

A. Baden, O. Baron, A. Belloni, S.C. Eno, Y. Feng, N.J. Hadley, S. Jabeen, G.Y. Jeng, R.G. Kellogg, J. Kunkle, A.C. Mignerey, S. Nabili, F. Ricci-Tam, M. Seidel, Y.H. Shin, A. Skuja, S.C. Tonwar, K. Wong

Massachusetts Institute of Technology, Cambridge, U.S.A.

D. Abercrombie, B. Allen, A. Baty, R. Bi, S. Brandt, W. Busza, I.A. Cali, M. D'Alfonso,

G. Gomez Ceballos, M. Goncharov, P. Harris, D. Hsu, M. Hu, M. Klute, D. Kovalskyi, Y.-J. Lee, P.D. Luckey, B. Maier, A.C. Marini, C. Mcginn, C. Mironov, S. Narayanan, X. Niu, C. Paus, D. Rankin, C. Roland, G. Roland, Z. Shi, G.S.F. Stephans, K. Sumorok,

K. Tatar, D. Velicanu, J. Wang, T.W. Wang, B. Wyslouch

University of Minnesota, Minneapolis, U.S.A.

R.M. Chatterjee, A. Evans, S. Guts, P. Hansen, J. Hiltbrand, Sh. Jain, Y. Kubota, Z. Lesko, J. Mans, R. Rusack, M.A. Wadud

University of Mississippi, Oxford, U.S.A.

J.G. Acosta, S. Oliveros 
University of Nebraska-Lincoln, Lincoln, U.S.A.

K. Bloom, D.R. Claes, C. Fangmeier, L. Finco, F. Golf, R. Kamalieddin, I. Kravchenko, J.E. Siado, G.R. Snow ${ }^{\dagger}$, B. Stieger, W. Tabb

State University of New York at Buffalo, Buffalo, U.S.A.

G. Agarwal, C. Harrington, I. Iashvili, A. Kharchilava, C. McLean, D. Nguyen, A. Parker, J. Pekkanen, S. Rappoccio, B. Roozbahani

Northeastern University, Boston, U.S.A.

G. Alverson, E. Barberis, C. Freer, Y. Haddad, A. Hortiangtham, G. Madigan, B. Marzocchi, D.M. Morse, T. Orimoto, L. Skinnari, A. Tishelman-Charny, T. Wamorkar, B. Wang, A. Wisecarver, D. Wood

Northwestern University, Evanston, U.S.A.

S. Bhattacharya, J. Bueghly, T. Gunter, K.A. Hahn, N. Odell, M.H. Schmitt, K. Sung, M. Trovato, M. Velasco

University of Notre Dame, Notre Dame, U.S.A.

R. Bucci, N. Dev, R. Goldouzian, M. Hildreth, K. Hurtado Anampa, C. Jessop, D.J. Karmgard, K. Lannon, W. Li, N. Loukas, N. Marinelli, I. Mcalister, F. Meng, C. Mueller, Y. Musienko ${ }^{36}$, M. Planer, R. Ruchti, P. Siddireddy, G. Smith, S. Taroni, M. Wayne, A. Wightman, M. Wolf, A. Woodard

The Ohio State University, Columbus, U.S.A.

J. Alimena, B. Bylsma, L.S. Durkin, S. Flowers, B. Francis, C. Hill, W. Ji, A. Lefeld, T.Y. Ling, B.L. Winer

\section{Princeton University, Princeton, U.S.A.}

G. Dezoort, P. Elmer, J. Hardenbrook, N. Haubrich, S. Higginbotham, A. Kalogeropoulos,

S. Kwan, D. Lange, M.T. Lucchini, J. Luo, D. Marlow, K. Mei, I. Ojalvo, J. Olsen, C. Palmer, P. Piroué, J. Salfeld-Nebgen, D. Stickland, C. Tully, Z. Wang

University of Puerto Rico, Mayaguez, U.S.A.

S. Malik, S. Norberg

Purdue University, West Lafayette, U.S.A.

A. Barker, V.E. Barnes, S. Das, L. Gutay, M. Jones, A.W. Jung, A. Khatiwada, B. Mahakud, D.H. Miller, G. Negro, N. Neumeister, C.C. Peng, S. Piperov, H. Qiu, J.F. Schulte, J. Sun, F. Wang, R. Xiao, W. Xie

Purdue University Northwest, Hammond, U.S.A.

T. Cheng, J. Dolen, N. Parashar

Rice University, Houston, U.S.A.

U. Behrens, K.M. Ecklund, S. Freed, F.J.M. Geurts, M. Kilpatrick, Arun Kumar, W. Li, B.P. Padley, R. Redjimi, J. Roberts, J. Rorie, W. Shi, A.G. Stahl Leiton, Z. Tu, A. Zhang 
University of Rochester, Rochester, U.S.A.

A. Bodek, P. de Barbaro, R. Demina, J.L. Dulemba, C. Fallon, T. Ferbel, M. Galanti, A. Garcia-Bellido, O. Hindrichs, A. Khukhunaishvili, E. Ranken, R. Taus

Rutgers, The State University of New Jersey, Piscataway, U.S.A.

B. Chiarito, J.P. Chou, A. Gandrakota, Y. Gershtein, E. Halkiadakis, A. Hart, M. Heindl,

E. Hughes, S. Kaplan, I. Laflotte, A. Lath, R. Montalvo, K. Nash, M. Osherson, H. Saka,

S. Salur, S. Schnetzer, S. Somalwar, R. Stone, S. Thomas

University of Tennessee, Knoxville, U.S.A.

H. Acharya, A.G. Delannoy, G. Riley, S. Spanier

Texas A\&M University, College Station, U.S.A.

O. Bouhali ${ }^{76}$, M. Dalchenko, M. De Mattia, A. Delgado, S. Dildick, R. Eusebi, J. Gilmore,

T. Huang, T. Kamon ${ }^{77}$, S. Luo, S. Malhotra, D. Marley, R. Mueller, D. Overton, L. Perniè,

D. Rathjens, A. Safonov

Texas Tech University, Lubbock, U.S.A.

N. Akchurin, J. Damgov, F. De Guio, S. Kunori, K. Lamichhane, S.W. Lee, T. Mengke, S. Muthumuni, T. Peltola, S. Undleeb, I. Volobouev, Z. Wang, A. Whitbeck

Vanderbilt University, Nashville, U.S.A.

S. Greene, A. Gurrola, R. Janjam, W. Johns, C. Maguire, A. Melo, H. Ni, K. Padeken, F. Romeo, P. Sheldon, S. Tuo, J. Velkovska, M. Verweij

University of Virginia, Charlottesville, U.S.A.

M.W. Arenton, P. Barria, B. Cox, G. Cummings, R. Hirosky, M. Joyce, A. Ledovskoy, C. Neu, B. Tannenwald, Y. Wang, E. Wolfe, F. Xia

Wayne State University, Detroit, U.S.A.

R. Harr, P.E. Karchin, N. Poudyal, J. Sturdy, P. Thapa

University of Wisconsin - Madison, Madison, WI, U.S.A.

T. Bose, J. Buchanan, C. Caillol, D. Carlsmith, S. Dasu, I. De Bruyn, L. Dodd, F. Fiori, C. Galloni, B. Gomber ${ }^{78}$, H. He, M. Herndon, A. Hervé, U. Hussain, P. Klabbers, A. Lanaro, A. Loeliger, K. Long, R. Loveless, J. Madhusudanan Sreekala, D. Pinna, T. Ruggles, A. Savin, V. Sharma, W.H. Smith, D. Teague, S. Trembath-reichert, N. Woods

$\dagger$ : Deceased

1: Also at Vienna University of Technology, Vienna, Austria

2: Also at IRFU, CEA, Université Paris-Saclay, Gif-sur-Yvette, France

3: Also at Universidade Estadual de Campinas, Campinas, Brazil

4: Also at Federal University of Rio Grande do Sul, Porto Alegre, Brazil

5: Also at UFMS, Nova Andradina, Brazil

6: Also at Universidade Federal de Pelotas, Pelotas, Brazil

7: Also at Université Libre de Bruxelles, Bruxelles, Belgium

8: Also at University of Chinese Academy of Sciences, Beijing, China 
9: Also at Institute for Theoretical and Experimental Physics named by A.I. Alikhanov of NRC 'Kurchatov Institute', Moscow, Russia

10: Also at Joint Institute for Nuclear Research, Dubna, Russia

11: Also at Ain Shams University, Cairo, Egypt

12: Also at Zewail City of Science and Technology, Zewail, Egypt

13: Also at Purdue University, West Lafayette, U.S.A.

14: Also at Université de Haute Alsace, Mulhouse, France

15: Also at Erzincan Binali Yildirim University, Erzincan, Turkey

16: Also at CERN, European Organization for Nuclear Research, Geneva, Switzerland

17: Also at RWTH Aachen University, III. Physikalisches Institut A, Aachen, Germany

18: Also at University of Hamburg, Hamburg, Germany

19: Also at Brandenburg University of Technology, Cottbus, Germany

20: Also at Institute of Physics, University of Debrecen, Debrecen, Hungary, Debrecen, Hungary

21: Also at Institute of Nuclear Research ATOMKI, Debrecen, Hungary

22: Also at MTA-ELTE Lendület CMS Particle and Nuclear Physics Group, Eötvös Loránd University, Budapest, Hungary, Budapest, Hungary

23: Also at IIT Bhubaneswar, Bhubaneswar, India, Bhubaneswar, India

24: Also at Institute of Physics, Bhubaneswar, India

25: Also at Shoolini University, Solan, India

26: Also at University of Visva-Bharati, Santiniketan, India

27: Also at Isfahan University of Technology, Isfahan, Iran

28: Now at INFN Sezione di Bari ${ }^{a}$, Università di Bari ${ }^{b}$, Politecnico di Bari ${ }^{c}$, Bari, Italy

29: Also at Italian National Agency for New Technologies, Energy and Sustainable Economic Development, Bologna, Italy

30: Also at Centro Siciliano di Fisica Nucleare e di Struttura Della Materia, Catania, Italy

31: Also at Scuola Normale e Sezione dell'INFN, Pisa, Italy

32: Also at Riga Technical University, Riga, Latvia, Riga, Latvia

33: Also at Malaysian Nuclear Agency, MOSTI, Kajang, Malaysia

34: Also at Consejo Nacional de Ciencia y Tecnología, Mexico City, Mexico

35: Also at Warsaw University of Technology, Institute of Electronic Systems, Warsaw, Poland

36: Also at Institute for Nuclear Research, Moscow, Russia

37: Now at National Research Nuclear University 'Moscow Engineering Physics Institute' (MEPhI), Moscow, Russia

38: Also at St. Petersburg State Polytechnical University, St. Petersburg, Russia

39: Also at University of Florida, Gainesville, U.S.A.

40: Also at Imperial College, London, United Kingdom

41: Also at P.N. Lebedev Physical Institute, Moscow, Russia

42: Also at California Institute of Technology, Pasadena, U.S.A.

43: Also at Budker Institute of Nuclear Physics, Novosibirsk, Russia

44: Also at Faculty of Physics, University of Belgrade, Belgrade, Serbia

45: Also at Università degli Studi di Siena, Siena, Italy

46: Also at INFN Sezione di Pavia ${ }^{a}$, Università di Pavia ${ }^{b}$, Pavia, Italy, Pavia, Italy

47: Also at National and Kapodistrian University of Athens, Athens, Greece

48: Also at Universität Zürich, Zurich, Switzerland

49: Also at Stefan Meyer Institute for Subatomic Physics, Vienna, Austria, Vienna, Austria

50: Also at Burdur Mehmet Akif Ersoy University, BURDUR, Turkey

51: Also at Adiyaman University, Adiyaman, Turkey

52: Also at Şırnak University, Sirnak, Turkey 
53: Also at Tsinghua University, Beijing, China

54: Also at Beykent University, Istanbul, Turkey, Istanbul, Turkey

55: Also at Istanbul Aydin University, Istanbul, Turkey

56: Also at Mersin University, Mersin, Turkey

57: Also at Piri Reis University, Istanbul, Turkey

58: Also at Gaziosmanpasa University, Tokat, Turkey

59: Also at Ozyegin University, Istanbul, Turkey

60: Also at Izmir Institute of Technology, Izmir, Turkey

61: Also at Marmara University, Istanbul, Turkey

62: Also at Kafkas University, Kars, Turkey

63: Also at Istanbul Bilgi University, Istanbul, Turkey

64: Also at Hacettepe University, Ankara, Turkey

65: Also at Vrije Universiteit Brussel, Brussel, Belgium

66: Also at School of Physics and Astronomy, University of Southampton, Southampton, United Kingdom

67: Also at IPPP Durham University, Durham, United Kingdom

68: Also at Monash University, Faculty of Science, Clayton, Australia

69: Also at Bethel University, St. Paul, Minneapolis, U.S.A., St. Paul, U.S.A.

70: Also at Karamanoğlu Mehmetbey University, Karaman, Turkey

71: Also at Vilnius University, Vilnius, Lithuania

72: Also at Bingol University, Bingol, Turkey

73: Also at Georgian Technical University, Tbilisi, Georgia

74: Also at Sinop University, Sinop, Turkey

75: Also at Mimar Sinan University, Istanbul, Istanbul, Turkey

76: Also at Texas A\&M University at Qatar, Doha, Qatar

77: Also at Kyungpook National University, Daegu, Korea, Daegu, Korea

78: Also at University of Hyderabad, Hyderabad, India 